University of Wollongong

Research Online

Faculty of Engineering and Information

Faculty of Engineering and Information

Sciences - Papers: Part A

Sciences

$1-1-2016$

\title{
Antenna selection strategies for MIMO-OFDM wireless systems: an energy- efficiency perspective
}

Ngoc Phuc Le

University of Wollongong, pnl750@uowmail.edu.au

Farzad Safaei

University of Wollongong, farzad@uow.edu.au

Le Chung Tran

University of Wollongong, Ictran@uow.edu.au

Follow this and additional works at: https://ro.uow.edu.au/eispapers

Part of the Engineering Commons, and the Science and Technology Studies Commons

Research Online is the open access institutional repository for the University of Wollongong. For further information contact the UOW Library: research-pubs@uow.edu.au 


\title{
Antenna selection strategies for MIMO-OFDM wireless systems: an energy- efficiency perspective
}

\author{
Abstract \\ In this paper, we investigate antenna selection strategies for MIMO-OFDM wireless systems from an \\ energy efficiency perspective. We first derive closed-form expressions of the energy efficiency and the \\ energy efficiency- spectral efficiency (EE-SE) trade-off in conventional antenna selection MIMO-OFDM \\ systems. The obtained results show that these systems suffer from a significant loss in energy efficiency. \\ To achieve a better energy-efficiency performance, we propose an adaptive antenna selection method \\ where both the number of active RF (radio frequency) chains and the antenna indices are selected \\ depending on the channel condition. This selection scheme could be implemented by an exhaustive \\ search technique for a small number of antennas. Moreover, we develop a greedy algorithm that achieves \\ a near-optimal performance with much lower complexity compared to the (optimal) exhaustive search \\ method when the number of antennas is large. In addition, the efficacy of power loading across \\ subcarriers for improved energy efficiency in the conventional and proposed antenna selection MIMO- \\ OFDM systems is considered. Monte-Carlo simulation results are provided to validate our analyses.
}

\section{Keywords}

perspective, energy, systems, wireless, strategies, efficiency, selection, antenna, mimo, ofdm

Disciplines

Engineering | Science and Technology Studies

\section{Publication Details}

N. Phuc. Le, F. Safaei \& L. Chung. Tran, "Antenna selection strategies for MIMO-OFDM wireless systems: an energy-efficiency perspective," IEEE Transactions on Vehicular Technology, vol. 65, (4) pp. 2048-2062, 2016. 


\title{
Antenna Selection Strategies for MIMO-OFDM Wireless Systems: An Energy-Efficiency Perspective
}

Ngoc Phuc Le, Student Member, IEEE, Farzad Safaei, Senior Member, IEEE, and Le Chung Tran, Member, IEEE

\begin{abstract}
In this paper, we investigate antenna selection strategies for MIMO-OFDM wireless systems from an energy efficiency perspective. We first derive closed-form expressions of the energy efficiency and the energy efficiency- spectral efficiency (EE-SE) trade-off in conventional antenna selection MIMO-OFDM systems. The obtained results show that these systems suffer from a significant loss in energy-efficiency. To achieve a better energy-efficiency performance, we propose an adaptive antenna selection method where both the number of active RF (radio frequency) chains and the antenna indices are selected depending on the channel condition. This selection scheme could be implemented by an exhaustive search technique for a small number of antennas. Moreover, we develop a greedy algorithm that achieves a nearoptimal performance with much lower complexity compared to the (optimal) exhaustive search method when the number of antennas is large. In addition, the efficacy of power loading across subcarriers for improved energy efficiency in the conventional and proposed antenna selection MIMO-OFDM systems is considered. Monte-Carlo simulation results are provided to validate our analyses.
\end{abstract}

\section{Index Terms}

Antenna selection, energy efficiency, MIMO,OFDM systems.

\section{INTRODUCTION}

Recent years have seen increasing demands for high speed wireless communications. Besides, reducing energy consumption in wireless networks is of significant interest among academic and industrial researchers. This is due the fact that there are rising energy costs and carbon footprint of operating wireless networks with an increasing number of customers [1]. Consequently, energy efficiency, which is conventionally defined as the number of transmitted information bits per unit energy (bits/Joule), needs to be considered as one of the key design metrics for future networks [2], [3]. The improvement of energy-efficiency in wireless systems could be tackled at the component level (e.g., improve power amplifier efficiency), link level (e.g., discontinuous transmission and

The authors are with the School of Electrical, Computer and Telecommunications Engineering, University of Wollongong, Australia (e-mails:\{pnl750, farzad, lctran\}@uow.edu.au).

This work was presented in part at the $17^{\text {th }}$ International Symposium on Wireless Personal Multimedia Communications (WPMC), Sydney, Australia, Sept. 7-10, 2014. 
sleep modes), or network level (e.g., the layout of networks and their management) [3], [4].

A combination of MIMO (multi-input multi-output) techniques and OFDM (orthogonal frequency division multiplexing) has been considered as a key technique for high-speed wireless communications [5], [6]. This is due to the fact that OFDM transmission offers high spectral efficiency and robustness against intersymbol interference (ISI) in multipath fading channels. Meanwhile, MIMO techniques significantly increase data rate and/or link reliability. Specifically, the ergodic capacity of MIMO systems over fading channels is shown to increase linearly with the minimum of the number of transmit and receive antennas [7]. In fact, MIMO-OFDM has been adopted in current and future standards, such as WiMAX (Worldwide Interoperability for Microwave Access) IEEE 802.16m, WLAN (Wireless Local Area Network) IEEE 802.11n, and 3GPP LTE (Long Term Evolution)/LTE-Advanced [6]. Among various MIMO schemes, antenna selection appears to be a promising approach for OFDM systems. In antenna selection, only a subset of antennas is selected (subject to a given selection criterion) for transmissions. Therefore, this technique requires a low implementation cost and small amount of feedback information, compared to other beamforming or precoding techniques [8], [9]. Owing to these advantageous properties, antenna selection has been considered for the uplink of 4G LTE-Advanced [10].

In general, there are two fundamental approaches for the deployment of antenna selection in OFDM systems, namely, bulk selection (i.e., choosing the same antennas for all subcarriers) [11][13] and per-subcarrier selection (i.e., selecting antennas independently for each subcarrier) [11], [14]. The main benefit of the latter over the former is that a larger capacity and/or better error performance can be achieved by exploiting the frequency-selective nature of the fading channels [11]. However, the per-subcarrier selection scheme needs a larger number of radio frequency (RF) chains than bulk selection. Beside these two selection methods, a combined selection scheme has been considered recently in [15]-[17]. This selection scheme combines the bulk selection and persubcarrier approaches. However, to the best of our knowledge, all these works only investigated antenna selection OFDM systems from either capacity or error-performance perspective, for example, analysing diversity gain and coding gain [11], [15]-[17], measuring capacity [13], or evaluating error performance [12], [14].

Antenna selection is traditionally considered for improved capacity and/or error-performance. Recently, some research works have investigated energy efficiency in antenna selection singlecarrier systems [18]-[20]. In [18], the authors jointly optimized the transmit power and the number of active antennas to maximize energy efficiency. This work examined single data stream MIMO systems, while energy-efficiency in multi-stream antenna selection MIMO single-carrier systems was studied in [19]. Transmit antenna selection with a large number of equipped antennas at the 
transmitter was considered in [20]. In this study, the authors analysed the energy efficiency in a large-scale array regime and proposed antenna selection algorithms to improve energy efficiency. A large-scale distributed antenna system (L-DAS) that considered antenna selection for improved energy-efficiency was also proposed in [21]. However, we note that these works only consider single-carrier systems, and an extension to antenna selection OFDM systems is not straightforward. The main reason is that there are several approaches for OFDM systems as mentioned before. From an energy-efficiency perspective, it can be seen that each antenna selection approach possesses both advantages and disadvantages. Specifically, per-subcarrier selection achieves better capacity than bulk selection and combined selection at a cost of higher power consumption due to the requirement of multiple active RF chains. These critical issues, which are pertinent in the setting of OFDM systems, do not arise in single-carrier antenna selection systems. Hence, they have not been considered so far. In addition, some recent works on energy-efficient MIMO-OFDM systems, e.g., [22], [23], focused only on spatial multiplexing MIMO schemes, which did not address the above concerns. Therefore, a study is required to evaluate energy efficiency of antenna selection MIMOOFDM systems. Note that in [24], we investigated energy-efficiency in per-subcarrier antenna subset selection OFDM systems with the objective of peak-power reduction. However, [24] only examined the per-subcarrier selection system from a viewpoint of power-amplifier efficiency, which is not the focus of the present work.

To the best of our knowledge, there is no comprehensive study about the efficacy of antenna selection schemes in the context of OFDM systems from an energy-efficiency viewpoint in the literature. Consequently, it is not clear if the existing antenna selection approaches (e.g., bulk selection, per-subcarrier selection, and combined selection schemes) are optimal in terms of energyefficiency. Motivated by this, in this paper, we investigate energy-efficiency in MIMO-OFDM systems with several antenna selection schemes. The main contributions of this work are summarized as follows.

i) Energy efficiency in conventional antenna selection MIMO-OFDM systems is analysed for the first time. In particular, we derive closed-form expressions of the energy efficiency and the EE-SE trade-off in these systems. Our results show that the conventional antenna selection systems are not effective with respect to energy efficiency.

ii) An adaptive antenna selection approach is proposed to improve energy efficiency in MIMOOFDM systems. In this method, both the number of active RF chains and the antenna indices are selected to maximize energy efficiency. We also show that the proposed adaptive selection scheme achieves better EE-SE trade-off compared to the existing selection schemes.

iii) A greedy algorithm to implement the proposed adaptive selection method is developed. This algorithm can attain near-optimal energy-efficiency while requiring much lower complexity 
compared to that with the optimal exhaustive search method, which is important when a number of antennas is large.

iv) Efficacy of power loading across subcarriers in several antenna selection MIMO-OFDM systems is evaluated from an energy-efficiency perspective. Our results reveal that power loading can improve energy efficiency in the low signal-to-noise ratio (SNR) region. Also, its effectiveness depends on particular antenna selection schemes.

v) Impacts of a comparison between the transmit power and the circuit power consumption, types of antenna selection criteria, the number of equipped antennas, and spatial correlation, on the energy efficiency in the conventional and proposed systems are numerically evaluated.

The remainder of the paper is organized as follows. In Section II, an antenna selection MIMOOFDM system model and an energy-efficiency metric are described. In Section III, we analyse energy-efficiency in the MIMO-OFDM systems deploying conventional antenna selection approaches. In Section IV, we propose an adaptive antenna selection method to improve energyefficiency. In Section V, power loading across subcarriers in antenna selection MIMO-OFDM systems is considered. In Section VI, we provide some simulation results and perform in-depth analyses of energy-efficiency achieved in the considered systems. Finally, Section VII concludes the paper.

Notation: Throughout this paper, a bold letter denotes a vector or matrix, whereas an italic letter denotes a variable. (. $)^{T},(.)^{H}, \varepsilon\{$.$\} , and \|.\|^{2}$ indicate transpose, Hermitian transpose, expectation, and a squared norm, respectively. Also, $\mathrm{C}_{a}^{b}=b ! / a !(b-a)$ ! denotes the binomial coefficient.

\section{ANTENNA SELECTION MIMO-OFDM WIRELESS SySTEMS}

\section{A. System Model}

We consider a point-to-point MIMO-OFDM system with $K$ subcarriers, $n_{T}$ transmit antennas, and $n_{R}$ receive antennas. The number of equipped transmit RF chains is $n_{R F}, n_{R F} \leq n_{T}$. A simplified block diagram of the system is shown in Fig. $1^{1}$. At the transmitter, the input data stream is mapped onto a unit-energy $M$-QAM ( $M$-ary quadrature amplitude modulation) or $M$-PSK $(M$-ary phase shift keying) constellation. The subcarrier allocation block takes in a data frame of $\mathbf{u}=[u(0), u(1), \ldots, u(K-1)]$, and then allocates the data symbol $u(k), 0 \leq k \leq K-1$, to the selected antenna, denoted as $\hat{i_{k}}$, associated with the $k^{\text {th }}$ subcarrier. Thus, only one element in a transmit

1 This system model is used for all the considered antenna selection methods in this paper. The association between RF chains and transmit antennas in each selection method is mentioned in Section III.A and Section IV. 


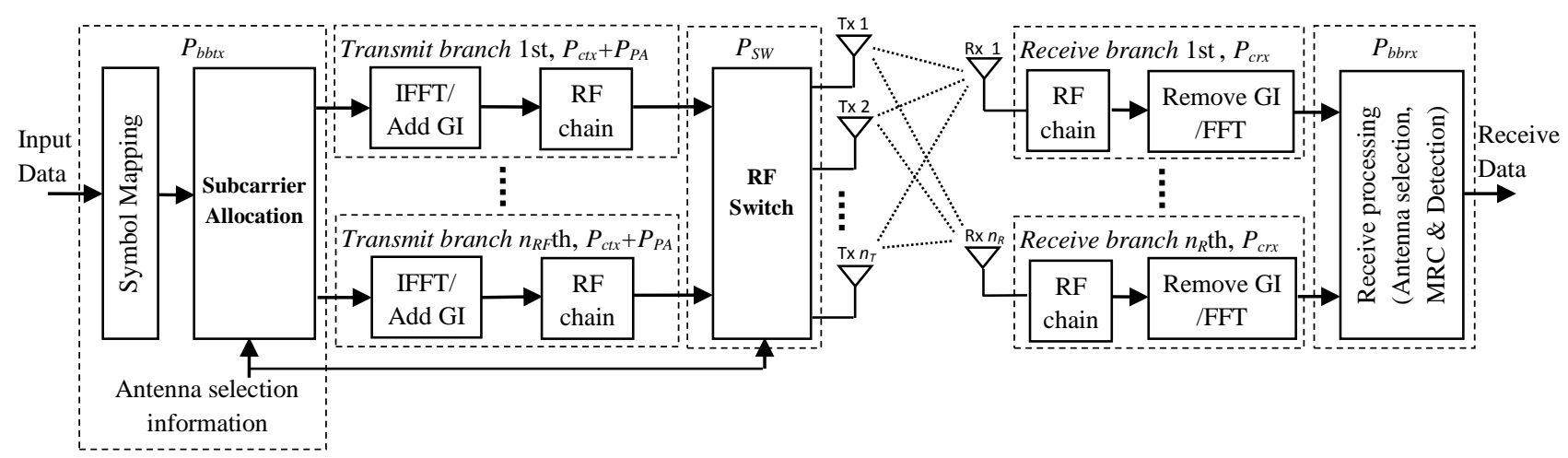

Fig. 1. A simplified block diagram of an antenna selection MIMO-OFDM wireless system.

vector $\mathbf{x}(k)=\left[x_{1}(k), x_{2}(k), \ldots, x_{n_{T}}(k)\right]^{T}$ is assigned the data symbol, whereas the others are zero ${ }^{2}$. The output sequences from the subcarrier allocation block are then fed into $K$-point IFFT (inverse fast Fourier transform) blocks. Each time-domain OFDM signal is then added with a guard interval (GI) before being transmitted via its corresponding transmit antenna. Note that the transmit branch corresponding to the output of the subcarrier allocation block that is not allocated any data symbol is turned off to save energy.

At the receiver, the received signal at each antenna is fed into the FFT block after the GI is removed. The received signal in the frequency domain corresponding to the $k^{\text {th }}$ subcarrier can be expressed as [5]

$$
\mathbf{y}(k)=\sqrt{P_{t}} \mathbf{H}(k) \mathbf{x}(k)+\mathbf{n}(k)=\sqrt{P_{t}} \mathbf{h}_{\bar{i}_{k}}(k) u(k)+\mathbf{n}(k),
$$

where $\mathbf{H}(k)$ denotes the subchannel matrix associated with the $k^{\text {th }}$ subcarrier where its entries are denoted as $h_{j, i}, i=1,2, . ., n_{T}, j=1,2, . ., n_{R}, \mathbf{h}_{\hat{i}_{k}}(k)$ indicates the effective channel vector obtained by selecting the column of $\mathbf{H}(k)$ that is corresponding to the selected transmit antenna $\hat{i}_{k}$ on the $k^{\text {th }}$ subcarrier, and $P_{t}$ is an equal transmit power allocated to each data subcarrier. Note that the total transmit power in one OFDM symbol is $P_{T}=K P_{t}$. Also, vectors $\mathbf{y}(k)=\left[y_{1}(k), y_{2}(k), \ldots, y_{n_{R}}(k)\right]^{T}$ and $\mathbf{n}(k)=\left[n_{1}(k), n_{2}(k), \ldots, n_{n_{R}}(k)\right]^{T}$, where $y_{j}(k)$ and $n_{j}(k)$ denote the received signal and the noise at the $j^{\text {th }}$ receive antenna, respectively. Here, the noise is modeled as a Gaussian random variable with zero-mean and variance $\sigma_{n}^{2}$. Assume that the receiver uses an MRC (maximum ratio combining) method for signal detection, the detected signal at the $k^{\text {th }}$ subcarrier is given as [26]

\footnotetext{
${ }^{2}$ In this work, we are interested in one-to-one mapping between data symbol and transmit antenna as in this approach the post-processing SNR is maximized [25].
} 


$$
\begin{aligned}
z(k) & =\sqrt{P_{t}}\left\|\mathbf{h}_{\hat{i}_{k}}(k)\right\|^{2} u(k)+\mathbf{h}_{\hat{i}_{k}}^{H}(k) \mathbf{n}(k) \\
& =\sqrt{P_{t}} g_{\widehat{i}_{k}}(k) u(k)+\tilde{n}(k),
\end{aligned}
$$

where $g_{\hat{i}_{k}}(k):=\left\|\mathbf{h}_{\hat{i}_{k}}(k)\right\|^{2}$, and $\tilde{n}(k)$ is the effective noise (after MRC) with variance $g_{\hat{i}_{k}}(k) \sigma_{n}^{2}$. In this system, the instantaneous post-processing SNR (signal-to-noise ratio) associated with the $i^{\text {th }}$ transmit antenna and the $k^{\text {th }}$ subcarrier can be calculated as $^{3}$ [7]

$$
\rho_{i}(k)=\frac{P_{t}\left(g_{i}(k)\right)^{2}}{g_{i}(k) \sigma_{n}^{2}}=\frac{P_{t}}{\sigma_{n}^{2}} g_{i}(k):=\bar{\rho} g_{i}(k),
$$

where $\bar{\rho}=P_{t} / \sigma_{n}^{2}$.

With respect to an antenna selection operation, many selection criteria can be used in this system, such as maximizing SNR, maximizing capacity, or minimizing bit-error rate [8]. Details about these criteria associated with different antenna selection approaches are presented in Section III.A. In addition, given that this work focuses on analyzing energy efficiency achieved in antenna selection MIMO-OFDM systems, the following assumptions are adopted for simplicity.

A1. Channel state information (CSI) is available at both transmitter and receiver in TDD (timeduplex division) mode. Thus, the transmitter and receiver can determine the selected antenna indices by themselves. Note that channel estimation methods for antenna selection OFDM system were well investigated in the literature, e.g., [27] and [28]. In addition, several techniques to obtain CSI of all equipped antennas when only a few antennas are active were also considered in [29].

A2. Effects of power unbalance across transmit antennas is not considered. The issue of power unbalance arises when a large number of subcarriers are allocated to some particular antennas, which may cause problems with power amplifiers (i.e., affects system performance). One approach to deal with this issue is allocating the same number of data symbols to each transmit antenna. This can be accomplished by formulating a linear optimization for subcarrier allocation [14]. The readers are referred to [14] for further details.

\section{B. Energy-Efficiency Metric in Antenna Selection OFDM Systems}

To quantify the fundamental limits of the system, we consider an energy-efficiency (bits/Joule)

${ }^{3}$ In this paper, $i\left(i=1,2, \ldots, n_{T}\right)$ denotes the transmit antenna index. Meanwhile, $\hat{i}_{k}$ denotes the selected antenna corresponding to the $k^{\text {th }}$ subcarrier, i.e., $\hat{i}_{k}=\arg \max _{i=1,2, \ldots, n_{T}} c_{i}(k)$, where $c_{i}(k)$ is the associated cost metric. Thus, for the expressions, e.g., (3) or (5), the index $i$ is used as these expressions are evaluated for all transmit antennas before a selection decision is made. 
defined as a ratio between the achievable rate and the total power consumption [18]-[24], i.e.,

$$
E E=C / P_{\text {total }},
$$

where $C$ denotes the achievable rate per OFDM symbol (bits/s) and $P_{\text {total }}$ is the required total power consumption (watts). Let us denote $I_{i}(k)$ to be the instantaneous capacity (bits/s/Hz) associated with the $i^{\text {th }}$ transmit antenna and the $k^{\text {th }}$ subcarrier [7], [30], i.e.,

$$
I_{i}(k)=\log _{2}\left(1+\bar{\rho} g_{i}(k)\right), i=1,2, \ldots, n_{T} ; k=0,1, \ldots, K-1 .
$$

The achievable rate per OFDM symbol in this system is evaluated by [30]

$$
C=W \varepsilon_{\mathbf{H}}\left\{\frac{1}{K} \sum_{k=0}^{K-1} I_{\widehat{i}_{k}}(k)\right\},
$$

where $\varepsilon_{\mathbf{H}}\{$.$\} denotes an expectation operation over the fading channel distribution, W(\mathrm{~Hz})$ is the system bandwidth.

The total power consumption corresponding to one OFDM symbol is given as [31]

$$
P_{\text {total }}=n_{o n}\left(P_{P A}+P_{c t x}\right)+n_{R} P_{c r x}+P_{b b},
$$

where $n_{o n}$ is the number of active RF chains ${ }^{4}$ (i.e., the number of active transmit branches) at the transmitter, $P_{P A}$ is the power consumption by one power amplifier (PA), $P_{c t x}$ is the power consumption per transmit branch (excluding the associated PA), $P_{c r x}$ is the power consumption per receive branch, and $P_{b b}=P_{b b t x}+P_{b b r x}$ where $P_{b b t x}$ and $P_{b b r x}$ are the power consumption of several baseband processing units at the transmitter and receiver, respectively. These values are shown clearly in Fig.1. Note that as power and insertion losses caused by RF switch are negligible [10], we do not include it in (7) for simplicity. When there are $n_{o n}$ active transmit branches, the number of data symbols allocated per transmit antenna is $K / n_{o n}$. Thus, the total transmit power per antenna is $P_{t}\left(K / n_{o n}\right)$. Assume that the efficiency $\eta$ of a power amplifier is invariant to the power output level, we can express the power drawn from a DC source $P_{P A}$ as [32]

$$
P_{P A}=\frac{P_{t}\left(K / n_{o n}\right)}{\eta}=\frac{P_{T} / n_{o n}}{\eta},
$$

where $P_{T}=K P_{\mathrm{t}}$ is the actual total transmit power per OFDM symbol. Note that the above assumption of constant efficiency can be realized by using PA with dynamic power supply [33]. Therefore, (7) can be rewritten as

$$
P_{\text {total }}=P_{T} / \eta+n_{o n} P_{c t x}+n_{R} P_{c r x}+P_{b b} .
$$

\footnotetext{
${ }^{4}$ A transmit RF chain consists of digital-to-analog (DAC), mixer, filters, and power amplifier. A receive RF chain consists of low-noise amplifier (LNA), filters, mixer, intermediate frequency amplifier, and analog-to-digital (ADC). In addition, a transmit (receive) local oscillator is assumed to be shared among transmit (receive) RF chains.
} 
From (4), (6), and (9), we can rewrite the (average) energy efficiency metric as

$$
E E=\frac{W \varepsilon_{\mathbf{H}}\left\{\frac{1}{K} \sum_{k=0}^{K-1} I_{\hat{i}_{k}}(k)\right\}}{P_{T} / \eta+n_{o n} P_{c t x}+n_{R} P_{c r x}+P_{b b}} .
$$

This metric will be used to evaluate energy efficiency in different antenna selection OFDM systems in the next sections.

\section{ENERGY-EFFICIENCY ANALYSIS OF CONVENTIONAL ANTENNA SELECTIONS SCHEMES}

\section{A. Conventional Antenna Selection Schemes}

As mentioned in Section II.A, several selection criteria can be used for antenna selection schemes. For notational convenience, let $c_{i}(k)$ denote the cost associated with the selection of the $i^{\text {th }}$ antenna on the $k^{\text {th }}$ subcarrier. Then, we can express

$$
c_{i}(k)=\left\{\begin{array}{cl}
g_{i}(k) & \text { for maximum channel power gain criterion (i.e., maximumSNR) } \\
I_{i}(k) & \text { for a maximum capacity criterion } \\
-B E R_{i}(k) & \text { for a miminum error - rate criterion, }
\end{array}\right.
$$

where $B E R_{i}(k)$ is a bit-error rate, e.g., for a $M$-QAM modulation with Gray mapping [34]

$$
B E R_{i}(k)=\frac{\sqrt{M}-1}{\sqrt{M} \log _{2} \sqrt{M}} \operatorname{erfc}\left(\sqrt{\frac{3 \bar{\rho} g_{i}(k)}{2(M-1)}}\right),
$$

where $\operatorname{erfc}($.$) denotes a complementary error function. Note that a negative sign is added to B E R_{i}(k)$ in (11), as we aim to maximize the cost $c_{i}(k)$ for all cases.

As mentioned in the introduction, antenna selection for OFDM systems can be implemented on a per-subcarrier basis or for a whole OFDM symbol. In per-subcarrier selection, antennas are selected independently for each subcarrier (see Fig. 2b for illustration). Assuming that $n_{R F}=n_{T}$, the selected antenna associated with the $k^{\text {th }}$ subcarrier is determined by [11],[14]

$$
\widehat{i_{k}}=\arg \max _{i=1, \ldots, n_{T}} c_{i}(k)
$$

Note that in this scheme, RF chains are connected directly to transmit antennas. As all $n_{T}$ RF chains are active, the total power consumption is $P_{t o t a l}^{p e r}=P_{T} / \eta+n_{T} P_{c t x}+n_{R} P_{c r x}+P_{b b}$.

Unlike per-subcarrier selection, in a bulk selection approach, only one among $n_{T}$ available antennas is used to transmit data (see Fig.2a). The antenna that can attain the largest accumulated SNR (i.e., the largest accumulated channel power gain) across subcarriers is selected for all 


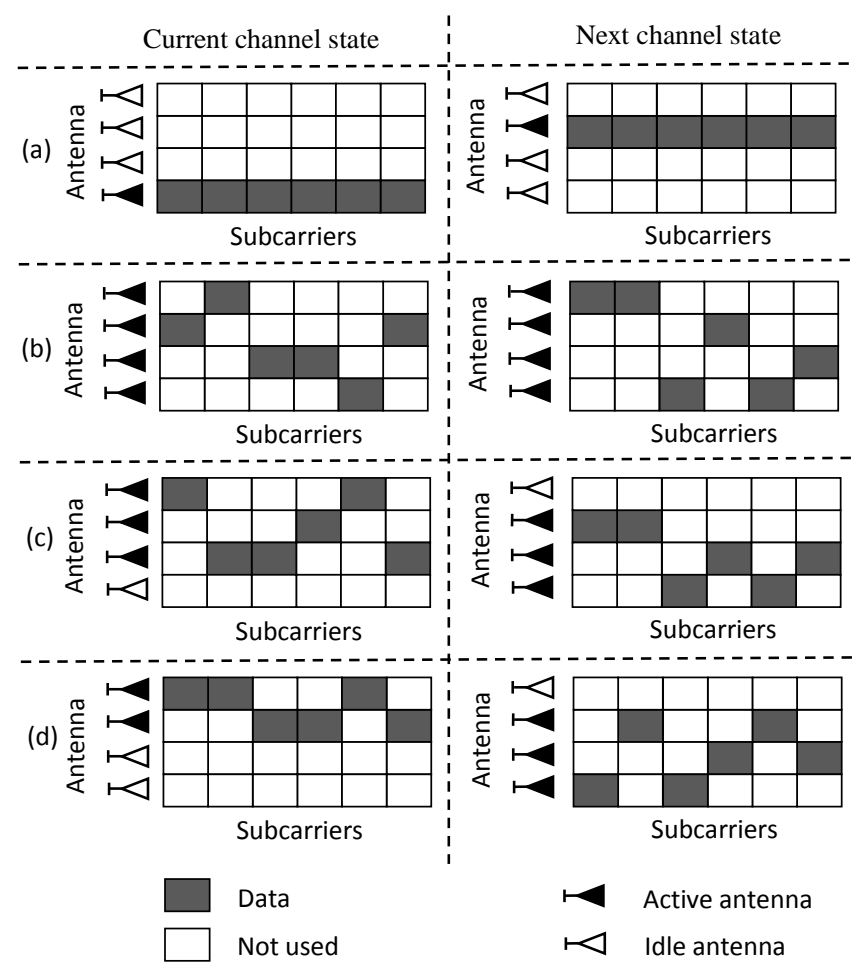

Fig. 2. Illustrations of antenna selection methods: (a) Bulk selection, (b) Per-subcarrier selection, (c) Combined selection, and (d) Proposed adaptive selection. $\left(n_{T}=4\right.$ and $\left.K=6\right)$.

subcarriers within one OFDM symbol, i.e., [11]-[13]

$$
\widehat{i}=\arg \max _{i=1, \ldots, n_{T}} \sum_{k=0}^{K-1} c_{i}(k)
$$

In this scheme, a transmit RF chain is connected to the selected antenna by means of a RF switch. As only one transmit RF chain is required, the total power consumption in this case is $P_{\text {total }}^{\text {bulk }}=P_{T} / \eta+P_{c t x}+n_{R} P_{c r x}+P_{b b}$.

Besides the two above fundamental approaches, a combined bulk selection and per-subcarrier selection scheme was considered in [15]-[17] for MIMO-OFDM systems where only $n_{R F}<n_{T} \mathrm{RF}$ chains are equipped (see Fig.2c). Accordingly, the system first selects a subset of $n_{R F}$ antennas and then performs per-subcarrier selection on this subset. A RF switch is required to connect $n_{R F} \mathrm{RF}$ chains to the subset of $n_{R F}$ selected antennas. Note that in this scheme, the number of active RF chains is $n_{o n}=n_{R F}$. As an exact analysis for this scheme is difficult due to the dependence between subsets of antennas, we will numerically evaluate this approach in Section VI.

\section{B. Analyses of Energy Efficiency in Conventional Antenna Selection MIMO-OFDM Systems}

In this subsection, we derive closed-form expressions of the energy efficiency and EE-SE tradeoff in antenna selection OFDM systems deploying per-subcarrier and bulk selection approaches. To the best of our knowledge, such results have not been available in the literature. For analytical 
simplicity, we assume that the fading coefficients $h_{j, i}(k)$ are i.i.d. (independent and identically distributed) Rayleigh random variables. This assumption is often adopted to analyze OFDM systems, see e.g., [11], [15]-[17].

\section{Per-Subcarrier Selection Scheme}

In an antenna selection OFDM system using a maximum SNR criterion (i.e., maximum channel power gain), assuming that subcarriers are independent, the capacity can be expressed as (cf. (6))

$$
C_{p e r}=W \varepsilon_{\mathbf{H}}\left\{\frac{1}{K} \sum_{k=0}^{K-1} I_{\hat{i}_{k}}(k)\right\}=W \varepsilon_{\mathbf{H}}\left\{\log _{2}\left(1+\bar{\rho} g_{\bar{i}_{k}}(k)\right)\right\}
$$

which can be evaluated at any subcarrier $k$. Therefore, the energy efficiency in this system is obtained as (cf. (10))

$$
E E_{p e r}=\frac{C_{p e r}}{P_{\text {total }}^{\text {per }}}=\frac{W \varepsilon_{\mathbf{H}}\left\{\log _{2}\left(1+\bar{\rho} g_{\widehat{i}_{k}}(k)\right)\right\}}{P_{T} / \eta+n_{T} P_{c t x}+n_{R} P_{c r x}+P_{b b}}
$$

To obtain a closed-form expression of (16), we need to derive an explicit expression for $\varepsilon_{\mathbf{H}}\left\{\log _{2}\left(1+\bar{\rho} g_{\widehat{i}_{k}}(k)\right)\right\}$. The final result is stated in the following theorem.

Theorem 1. A closed-form expression of the energy efficiency in per-subcarrier antenna selection MIMO-OFDM systems is given by

$$
E E_{\text {per }}=\frac{W n_{T}}{P_{\text {total }}^{\text {per }}\left(n_{R}-1\right) ! \ln 2} \sum_{u=0}^{n_{T}-1}\left\{(-1)^{u} \mathrm{C}_{u}^{n_{T}-1} \sum_{q=0}^{\left(n_{R}-1\right) u}\left(\alpha_{u, q} \frac{\left(n_{R}+q-1\right) !}{\bar{\rho}^{n_{R}+q}} e^{(u+1) / \rho} \sum_{s=1}^{n_{R}+q}\left(\frac{u+1}{\bar{\rho}}\right)^{-s} \Gamma\left(-n_{R}-q+s, \frac{u+1}{\bar{\rho}}\right)\right)\right\}
$$

where $\alpha_{u, q}$ denotes the multinomial coefficient, and $\Gamma(a, x)=\int_{x}^{+\infty} e^{-t} t^{a-1} d t$ is the incomplete gamma function.

Proof: The proof is given in Appendix A.

\section{Bulk Selection Scheme}

For a bulk antenna selection OFDM system, the capacity can be expressed as (cf. (6))

$$
C_{\text {bulk }}=W \varepsilon_{\mathbf{H}}\left\{\frac{1}{K} \sum_{k=0}^{K-1} I_{\hat{i}}(k)\right\}=W \varepsilon_{\mathbf{H}}\left\{\frac{1}{K} \sum_{k=0}^{K-1} \log _{2}\left(1+\bar{\rho} g_{\hat{i}}(k)\right)\right\}
$$

By using an approximation of $\log _{2}(1+x) \approx x \log _{2} e$, when $x$ is small, we can express the capacity at the low SNR region as

$$
C_{\text {bulk }} \approx W\left(\bar{\rho} \log _{2} e\right) \times \varepsilon_{\mathbf{H}}\left\{\frac{1}{K} \sum_{k=0}^{K-1} g_{\hat{i}}(k)\right\} .
$$


Consequently, the energy efficiency now becomes (cf. (10))

$$
E E_{\text {bulk }}=\frac{C_{\text {bulk }}}{P_{\text {total }}^{\text {bolk }}} \approx \frac{W\left(\bar{\rho} \log _{2} e\right) \times \varepsilon_{\mathbf{H}}\left\{\frac{1}{K} \sum_{k=0}^{K-1} g_{\widehat{i}}(k)\right\}}{P_{T} / \eta+P_{c t x}+n_{R} P_{c r x}+P_{b b}} .
$$

By calculating the expected value in the numerator of (20), we arrive at the following result.

Theorem 2. The energy efficiency in bulk antenna selection MIMO-OFDM systems in the low SNR regime is approximated as

$$
E E_{\text {bulk }} \approx \frac{W\left(\bar{\rho} \log _{2} e\right) n_{T}}{P_{\text {total }}^{\text {bulk }} K\left(n_{R} K-1\right) !} \sum_{u=0}^{n_{T}-1}\left\{(-1)^{u} C_{u}^{n_{T}-1} \sum_{t=0}^{\left(n_{R} K-1\right) u} \beta_{u, t}\left(n_{R} K+t\right) !(u+1)^{-n_{R} K-t-1}\right\},
$$

where $\beta_{u, t}$ denotes the multinomial coefficient.

Proof: The proof is given in Appendix B.

\section{Energy Efficiency-Spectral Efficiency Trade-off}

In this subsection, we derive closed-from expressions for the energy efficiency- spectral efficiency (EE-SE) trade-off. Recall that EE (bits/Joule) is defined in (4) as $E E=C / P_{\text {total }}$. Also, the spectral efficiency SE (bits/s/Hz) is calculated as $S E=C / W$, where $C$ (bits/s) is the capacity and $W$ $(\mathrm{Hz})$ is the system bandwidth. Thus, the relation between EE and SE can be expressed as [35]

$$
E E=\frac{C}{P_{\text {total }}}=\frac{W \times S E}{K P_{t} / \eta+n_{o n} P_{c t x}+n_{R} P_{c r x}+P_{b b}}=\frac{W \times S E}{(K / \eta) \times f^{-1}(S E)+n_{o n} P_{c t x}+n_{R} P_{c r x}+P_{b b}},
$$

where $f^{-1}: S E \in[0,+\infty) \mapsto P_{t} \in[0,+\infty)$ is the inverse function of SE. In what follows, we consider per-subcarrier and bulk selection schemes at the low SNR region for analytical simplicity.

In per-subcarrier selection, by using an approximation of $\log _{2}(1+x) \approx x \log _{2} e$, when $x$ is small, we can express the capacity at the low SNR region as (cf. (15))

$$
C_{p e r} \approx W\left(\bar{\rho} \log _{2} e\right) \times \varepsilon_{\mathbf{H}}\left\{g_{\bar{i}_{k}}(k)\right\}
$$

By performing similar calculations as in Appendix A, we have

$$
\varepsilon_{\mathbf{H}}\left\{g_{\hat{i}_{k}}(k)\right\}=\frac{n_{T}}{\left(n_{R}-1\right) !} \sum_{u=0}^{n_{T}-1}\left\{(-1)^{u} \mathrm{C}_{u}^{n_{T}-1} \sum_{q=0}^{\left(n_{R}-1\right) u} \alpha_{u, q}\left(n_{R}+q\right) !(u+1)^{-n_{R}-q-1}\right\}
$$

Thus, (23) can be rewritten as

$$
C_{p e r} \approx W\left(\bar{\rho} \log _{2} e\right) \times \frac{n_{T}}{\left(n_{R}-1\right) !} \sum_{u=0}^{n_{T}-1}\left\{(-1)^{u} \mathrm{C}_{u}^{n_{T}-1} \sum_{q=0}^{\left(n_{R}-1\right) u} \alpha_{u, q}\left(n_{R}+q\right) !(u+1)^{-n_{R}-q-1}\right\} .
$$


For notational convenience, let us denote

$$
\varphi=\frac{n_{T} \log _{2} e}{\left(n_{R}-1\right) ! \sigma_{n}^{2}} \sum_{u=0}^{n_{T}-1}\left\{(-1)^{u} \mathrm{C}_{u}^{n_{T}-1} \sum_{q=0}^{\left(n_{R}-1\right) u} \alpha_{u, q}\left(n_{R}+q\right) !(u+1)^{-n_{R}-q-1}\right\} .
$$

Also, recall that $\bar{\rho}=P_{t} / \sigma_{n}^{2}$.Then, we can express the capacity $C_{p e r}$ and spectral efficiency $S E_{p e r}$, respectively, as

$$
C_{p e r} \approx W \times P_{t} \times \varphi
$$

and

$$
S E_{p e r} \approx P_{t} \times \varphi
$$

From (22), (27), (28), and noting that $P_{T}=K P_{t}$, we arrive at the following result.

Proposition 1. The closed-form expression for the EE-SE trade-off in per-subcarrier antenna selection systems in the low SNR regime is approximated as

$$
E E_{p e r} \approx \frac{W \times S E_{p e r}}{(K / \eta \varphi) S E_{p e r}+n_{T} P_{c t x}+n_{R} P_{c r x}+P_{b b}} .
$$

For the bulk selection scheme, an expression for the EE-SE trade-off is given below.

Proposition 2. The closed-form expression for the EE-SE trade-off in bulk antenna selection systems in the low SNR regime can be approximated by

$$
E E_{b u l k} \approx \frac{W \times S E_{b u l k}}{(K / \eta \chi) S E_{b u l k}+P_{c t x}+n_{R} P_{c r x}+P_{b b}},
$$

where

$$
\chi=\frac{n_{T} \log _{2} e}{K\left(n_{R} K-1\right) ! \sigma_{n}^{2}} \sum_{u=0}^{n_{T}-1}\left\{(-1)^{u} \mathrm{C}_{u}^{n_{T}-1} \sum_{t=0}^{\left(n_{R} K-1\right) u} \beta_{u, t}\left(n_{R} K+t\right) !(u+1)^{-n_{R} K-t-1}\right\}
$$

Proof: The result is obtained based on (21) and (22).

\section{Numerical and Simulation Example}

To validate the analysis above, we run a simulation for the system with $n_{T}=4, n_{R}=1, K=16$, $W=1 \mathrm{MHz}, \eta=0.35$, and $P_{c t x}=P_{c r x}=P_{b b}=50 \mathrm{~mW}$. Note that although the number of subcarriers $K$ is small, they are assumed independent. Moreover, simulation results under more realistic parameters will be provided in Section VI. Figure 3 plots the energy efficiency versus the total transmit power $P_{T}$ at the low SNR regime. It can be seen that the analytical curves based on the analysis in the previous sections match the simulation curves. 


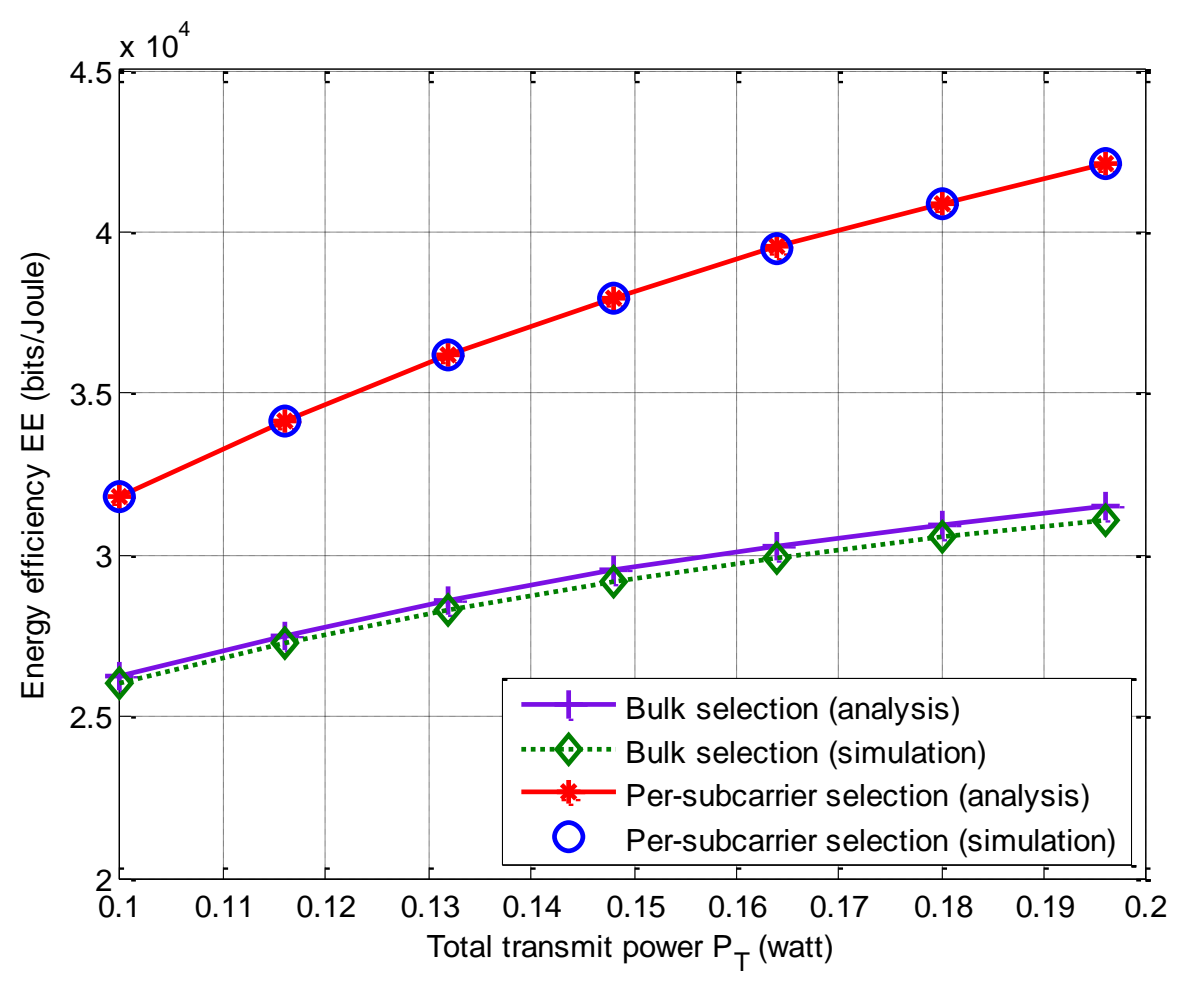

Fig. 3. Energy-efficiency in bulk selection and per-subcarrier selection: analysis vs. simulation.

\section{AdAPTIVE ANTENNA SELECTION FOR IMPROVED ENERGY-EFFICIENCY}

The average energy efficiency in a generic antenna selection MIMO-OFDM system is given in (10). It can be seen that the $E E$ value depends on many factors, including the actual transmit power, the power consumed by the electronics circuits (mainly RF chains), as well as the channel condition. When one antenna (i.e., one RF chain) is activated/deactivated, the system will achieve a higher/lower capacity. Meanwhile, the power consumption due to RF chains is increased/decreased. Consequently, whether the $E E$ value is increased or not depends on the changes of the capacity and consumed power. Given fixed power values of $P_{T}, P_{c t x}, P_{c r x}$, and $P_{b b}$, whether an antenna should be activated or deactivated for improved energy-efficiency depends on the channel condition. Based on these observations, we propose to improve the energy efficiency by adaptively selecting both the number of active RF chains $n_{o n}\left(1 \leq n_{o n} \leq n_{R F}\right)$ and the transmit antenna indices (see Fig. $2 \mathrm{~d}$ for illustration). In this adaptive scheme, $n_{\text {on }}$ active RF chains are connected to the subset of $n_{\text {on }}$ selected antennas via a RF switch. The adaptive selection can be implemented by either an exhaustive search or a low-complexity algorithm, which are described in detail below.

\section{A. Exhaustive Search Method}

When the number of transmit antennas $n_{T}$ is small, an exhaustive search method can be used to achieve an optimal antenna allocation. In particular, this method checks all possible subsets of antennas, and selects the subset that attains the highest energy efficiency value. Note that the 
1: Initial setting: A subset of unallocated subcarriers $\Omega_{0}=\{1,2, \ldots, K\}$,

A subset of available transmit antennas $S_{0}=\left\{1,2, \ldots, n_{T}\right\}$,

A subset of selected antennas $P_{0}=\{\varnothing\}$, and an energy-efficiency value $E E_{0}=0$.

2: Calculate the cost $c_{i}(k), \forall i \in S_{0}, \forall k \in \Omega_{0}$, using Eq. (11).

3: for $m=1: n_{R F}$ do

4: Calculate the accumulated cost across unallocated subcarriers (used to select antenna)

$$
\Psi_{i}^{(m)}=\sum_{k \in \Omega_{m-1}} c_{i}(k), i \in S_{m-1} .
$$

5: $\quad$ Select the antenna $\hat{i}(m)$ that satisfies $\hat{i}(m)=\arg \max _{i \in S_{m-1}} \Psi_{i}^{(m)}$.

6: $\quad$ Add $\hat{i}(m)$ to the subset of selected antennas, i.e., $P_{m}=\left\{P_{m-1}, \hat{i}(m)\right\}$.

7: $\quad$ Assign the selected antenna $\hat{i}(m)$ to the subcarriers $l$ that satisfy

$$
c_{\hat{i}(m)}(l)=\arg \max _{i \in S_{m-1}} c_{i}(l),
$$

i.e., $\hat{i}_{(m)}(l)=\hat{i}(m), \forall l \in \bar{\Omega}_{m}$, where $\bar{\Omega}_{m}$ is the subset of the allocated subcarriers $l$ in the $m^{\text {th }}$ loop, and $\hat{i}_{(m)}(l)$ is the selected antenna at the subcarrier $l$ in the $m^{\text {th }}$ loop.

8: Update the subset of unallocated subcarriers as $\Omega_{m}=\Omega_{m-1}-\bar{\Omega}_{m}$.

9: $\quad$ Select antennas in $P_{m}$ for the remain unallocated subcarriers via

$$
\hat{i}_{(m)}(\kappa)=\arg \max _{i \in P_{m}} c_{i}(\kappa), \forall \kappa \in \Omega_{m} .
$$

10: Calculate the accumulated instantaneous capacity (bits/s/Hz) corresponding to the subset $P_{m}$ (used to calculate the $E E$ value) (cf. (5))

$$
\bar{I}_{m}=\sum_{k=0}^{K-1} I_{\hat{i}_{(m)}(k)}(k) \text {. }
$$

11: Calculate the $E E$ value (cf. (10)):

$$
E E_{m}=(W / K) \times \bar{I}_{m} /\left(P_{T} / \eta+m P_{c t x}+n_{R} P_{c r x}+P_{b b}\right) .
$$

12: if $E E_{m}>E E_{0}$ do

13: $\quad E E_{0}=E E_{m}$,

14: $\quad P_{\text {select }} \leftarrow P_{m}$,

15: $\quad \hat{i}_{\text {select }}(k) \leftarrow \hat{i}_{(m)}(k), k=1,2, \ldots, K$.

16: end if

17: Update the subset of available antennas as $S_{m}=S_{0}-P_{m}$.

18: end for

19: The subset of selected antennas and the allocation pattern for the maximal $E E$ value are $P_{\text {select }}$ and $\hat{i}_{\text {select }}(k), k=1,2, \ldots, K$, respectively.

number of possible subsets is $\sum_{m=1}^{n_{R F}} C_{m}^{n_{T}}$, which incurs very high complexity if $n_{T}$ and/or $n_{R F}$ are large. Thus, a lower complexity method is preferred in this scenario.

\section{B. Low-Complexity Algorithm}

To realize the proposed adaptive selection method with low complexity, we develop a greedy selection algorithm described in Table I. This algorithm selects antennas in an incremental fashion and is based on the following principles:

P1. Given a subset $P_{m-1}$ consisting of $(m-1)$ selected antennas, the best antenna $\hat{i}(m)$ that is added to create the subset $P_{m}$ is the antenna that makes $P_{m}$ achieve the largest accumulated cost. 
TABLE II. COMPLEXITY COMPARISON $(K=64)$.

\begin{tabular}{|l|c|c|c|c|c|c|c|}
\hline Number of transmit antennas $n_{T}$ & 2 & 3 & 4 & 5 & 6 & 7 & 8 \\
\hline$\zeta_{\text {opt }}($ exhaustive search) & $3 K$ & $7 K$ & $15 K$ & $31 K$ & $63 K$ & $127 K$ & $255 K$ \\
\hline$\zeta_{\text {sub }}=K n_{T}\left(n_{T}+1\right) / 2$ (worst-case) & $3 K$ & $6 K$ & $10 K$ & $15 K$ & $21 K$ & $28 K$ & $36 K$ \\
\hline$\zeta_{\text {sub }}($ average) & $2.3 K$ & $4.1 K$ & $6.1 K$ & $8.4 K$ & $10.9 K$ & $13.6 K$ & $16.5 K$ \\
\hline$\zeta_{\text {opt }} / \zeta_{\text {sub }}($ average) & 1.3 & 1.7 & 2.5 & 3.7 & 5.8 & 9.3 & 15.5 \\
\hline
\end{tabular}

P2. If the cost $c_{\hat{i}(m)}(l)$ is the largest among the available antennas at the $l^{\text {th }}$ subcarrier, then the antenna $\hat{i}(m)$ is immediately selected for the $l^{\text {th }}$ subcarrier when this antenna is added to the subset $P_{m-1}$. Note that the value $c_{\tilde{i}(m)}(l)$ will be always taken as the cost on the $l^{\text {th }}$ subcarrier when measuring the accumulated cost for all $n_{R F}$ subsets $P_{m}, m=1,2, \ldots, n_{R F}$. Therefore, the cost corresponding to the $l^{\text {th }}$ subcarrier on the remaining available antennas will not be taken into account when evaluating the accumulated cost of these antennas. Consequently, the optimal antenna $\hat{i}_{m}$ mentioned in $\mathbf{P 1}$ is the one that has the largest accumulated cost calculated only over a subset of unallocated subcarriers.

\section{Complexity Evaluation}

With respect to a complexity comparison between the algorithm in Table I and the exhaustive search, we consider the number of allocation operations as a measure of complexity. In the exhaustive search, there are $\sum_{m=1}^{n_{R F}} C_{m}^{n_{T}}$ possible subsets, and each subset needs $K$ allocations for $K$ subcarriers. Thus, the number of allocations is $\zeta_{\text {opt }}=K \sum_{m=1}^{n_{R F}} C_{m}^{n_{T}}$. When $n_{R F}=n_{T}$, the value $\zeta_{\text {opt }}$ is given to $\zeta_{\text {opt }}=K \sum_{m=1}^{n_{T}} C_{m}^{n_{T}}=K\left(2^{n_{T}}-1\right)$. Meanwhile, in the proposed algorithm, the $m^{\text {th }}(m=1,2, \ldots$, $\left.n_{R F}\right)$ loop searches for $\left(n_{T}-m+1\right)$ subsets and performs $\left|\Omega_{m-1}\right|$ allocations for each subset. Here, $\left|\Omega_{m-1}\right|$ denotes the cardinality of the subset $\Omega_{m-1}$, i.e., $\left|\Omega_{m-1}\right| \leq K$. Therefore, the proposed algorithm requires only $\zeta_{s u b}=\sum_{m=1}^{n_{R F}}\left(n_{T}-m+1\right)\left|\Omega_{m-1}\right|$ allocations. A complexity comparison between the exhaustive search and algorithm methods based on numerical values is shown in Table II. In this table, the results are averaged over $10^{5}$ channel realizations. Details about other simulation parameters are provided in Section VI. The obtained results show that the algorithm attains very low complexity compared to the exhaustive search method.

Let us further consider the complexity of the proposed algorithm in the worst case, i.e., $\left|\Omega_{m-1}\right|=K, \forall m=1,2, \ldots, n_{R F}$. In this scenario, the number of allocations is $\zeta_{s u b}=K \sum_{m=1}^{n_{R F}}\left(n_{T}-m+1\right)$. When $n_{R F}=n_{T}$, we have $\zeta_{s u b}=K \sum_{m=1}^{n_{T}}\left(n_{T}-m+1\right)=K n_{T}\left(n_{T}+1\right) / 2$ (i.e., increase polynomially with 
TABLE III. NUMBER OF UNALLOCATED SUBCARRIERS $(K=64)$.

\begin{tabular}{|c|c|c|c|c|c|}
\hline$n_{T}$ & $\left|\Omega_{0}\right|$ & $\left|\Omega_{1}\right|$ & $\left|\Omega_{2}\right|$ & $\left|\Omega_{3}\right|$ & $\left|\Omega_{4}\right|$ \\
\hline 2 & $K$ & $0.34 K$ & & & \\
\hline 3 & $K$ & $0.46 K$ & $0.14 K$ & & \\
\hline 4 & $K$ & $0.52 K$ & $0.23 K$ & $0.07 K$ & \\
\hline 5 & $K$ & $0.56 K$ & $0.29 K$ & $0.12 K$ & $0.03 K$ \\
\hline
\end{tabular}

respect to $\left.n_{T}\right)$ compared to $\zeta_{\text {opt }}=K\left(2^{n_{T}}-1\right)$ (i.e., increase exponentially). Consequently, the proposed algorithm in Table I still incurs lower complexity, especially when $n_{T}$ is large. It is also worth mentioning that the value $\zeta_{s u b}$ (average) is smaller than $\zeta_{s u b}$ (worst-case) as the number of unallocated subcarriers $\left|\Omega_{m-1}\right|$ becomes much smaller after each loop as shown in Table III.

\section{POWER LOADING FOR ANTENNA SELECTION MIMO-OFDM SYSTEMS}

In the previous sections, we have considered the systems with equal power allocation across selected subcarriers, i.e., $P_{t, k}=P_{T} / K:=P_{t}, \forall k$. This equal power allocation may be required in systems where a very strict spectral mask applied on each subcarrier, e.g., multiband-OFDM ultrawideband (MB-OFDM UWB) [36]. However, if a spectral mask constraint on the $k^{\text {th }}$ subcarrier is $P_{k}^{\text {mask }}$, power loading across selected subcarriers, which means dynamic distribution of the available power among subcarriers, can be employed to further improve energy efficiency. This is because power loading can increase capacity in OFDM systems [37], which in turn improves energy efficiency (cf. (10)). Our formulation problem in this section is stated as follows: Suppose that the total transmit power is $P_{T}$, find the optimal allocated powers $\left\{P_{t, k}^{*}, k=0,1, \ldots, K-1\right\}$ that satisfy a spectral mask constraint so that the energy-efficiency in antenna selection MIMO-OFDM systems is maximized.

We assume that antennas are selected for all subcarriers based on a given selection scheme (e.g., bulk selection, per-subcarrier selection, combined selection, or adaptive selection scheme) that has been described in the previous sections. In what follows, we will derive the optimal allocation of powers $\left\{P_{t, k}^{*}, k=0,1, \ldots, K-1\right\}$. Recall that the channel power gain (after MRC) and the allocated power associated with the $k^{\text {th }}$ subcarrier are $g_{\hat{i}_{k}}(k)$ and $P_{t, k}$, respectively. Hence, the instantaneous energy efficiency can be expressed as (cf. (3), (5), (10))

$$
E E=\frac{(W / K) \times \sum_{k=0}^{K-1} \log _{2}\left(1+P_{t, k} g_{\hat{i}_{k}}(k) / \sigma_{n}^{2}\right)}{\sum_{k=0}^{K-1} P_{t, k} / \eta+n_{o n} P_{c t x}+n_{R} P_{c r x}+P_{b b}} .
$$


We aim to allocate powers $\left\{P_{t, k}\right\}$ such that the $E E$ value in (32) is maximized subject to the following constraints. The first constraint is that the power allocated on the $k^{\text {th }}$ subcarrier $P_{t, k}$ is not larger than the corresponding spectral mask $P_{k}^{\text {mask }}$, i.e.,

$$
0 \leq P_{t, k} \leq P_{k}^{\text {mask }}, k=0,1, \ldots, K-1 \text {. }
$$

The second constraint requires the total power allocated all over the subcarriers to be equal to $P_{T}$, i.e.,

$$
\sum_{k=0}^{K-1} P_{t, k}=P_{T}
$$

Note that this constraint guarantees a fair comparison among antenna selection schemes as our focus in this work is to determine which scheme can attain the highest $E E$ value given the same actual transmit power $P_{T}$. Due to the second constraint (34), the denominator of (32) is a constant with respect to $\left\{P_{t, k}\right\}$. Therefore, the power loading problem to maximize energy-efficiency can now be expressed as

$$
\begin{aligned}
& \max _{\left\{P_{t, k}\right\}} \sum_{k=0}^{K-1} \log _{2}\left(1+P_{t, k} g_{\hat{i}_{k}}(k) / \sigma_{n}^{2}\right) \\
& \text { s.t. } \quad 0 \leq P_{t, k} \leq P_{k}^{\text {mask }}, k=0,1, \ldots, K-1, \\
& \quad \sum_{k=0}^{K-1} P_{t, k}=P_{T} .
\end{aligned}
$$

It is obvious that (35) is a convex problem. Thus, its solution can be obtained as [38]

$$
P_{t, k}^{*}=\left[\vartheta-\frac{\sigma_{n}^{2}}{g_{\hat{i}_{k}}(k)}\right]_{0}^{P_{k}^{\text {mask }}}, k=0,1, \ldots, K-1,
$$

where $\vartheta>0$ is the water-level that is chosen to satisfy the total power constraint of $\sum_{k=0}^{K-1} P_{t, k}^{*}=P_{T}$, and $[x]_{a}^{b}$ denotes the Euclidean projection of $x$ on $[a, b]$, i.e., $[x]_{a}^{b}=\min (b, \max (x, a))$. Efficacy of power loading in associated with several antenna selection schemes that is evaluated numerically based on (26) will be discussed in Section VI.D ${ }^{5}$

Remark 1: Although power loading has been well studied for single-antenna OFDM systems, an investigation of power loading for antenna selection MIMO-OFDM systems is necessary. The

\footnotetext{
${ }^{5}$ We use the optimal water-filling (WF) solution of (36) in this work as our main purpose is to evaluate the efficacy of power loading in associated with several antenna selection schemes. However, it is worth noting that one can consider some approaches, e.g., cross-zero adjustment WF [39], that converge to the solution in (36) with a lower complexity.
} 
TABLE IV. SIMULATION PARAMETERS.

\begin{tabular}{|l|l|}
\hline \multicolumn{1}{|c|}{ Parameter } & \multicolumn{1}{|c|}{ Value } \\
\hline Carrier frequency & $f_{c}=2.4 \mathrm{GHz}$ \\
Bandwidth & $W=25 \mathrm{MHz}$ \\
Modulation & 4-QAM \\
Number of subcarriers & $K=64$ \\
Circuit powers & $P_{c t x}=150 \mathrm{~mW}, P_{c r x}=120 \mathrm{~mW}$, \\
& $P_{b b}=100 \mathrm{~mW}$ \\
Power amplifier efficiency & $35 \%$ \\
PSD of noise & $-174 \mathrm{dBm} / \mathrm{Hz}$ \\
Path-loss exponent & $\phi=4$ \\
Frequency-selective fading channel & IEEE $802.11 \mathrm{n}$ channel models \\
\hline
\end{tabular}

reason is that the effectiveness of power loading over equal allocation depends on a variation of channel power gains. Meanwhile, in antenna selection, statistical distribution properties of channel power gains corresponding to the selected subcarriers are altered due to a selection operation. Note that this characteristic does not occur in single-antenna OFDM systems. Therefore, it is interesting to know, from an energy-efficiency perspective, how effective power allocation is in the context of antenna selection in OFDM systems.

Remark 2: In this work, we perform power loading after antenna selection. The advantage of this approach is that it requires very low additional complexity. In fact, in this approach, the power loading operation (26) is performed only once at the transmitter only. Note that one can perform joint power loading and antenna selection. However, for a joint approach, as the allocated powers on subcarriers are involved in the calculation of antenna selection metrics, we need to perform power loading operation several times during an antenna selection process. Moreover, in a TDD mode, this joint method needs to be performed at both transmitter and receiver. This clearly introduces high complexity. An analysis of the joint approach as well as its performance-complexity trade-off is beyond the scope of this paper.

\section{SimUlation RESUlTS AND DisCUSSIONS}

In this section, we evaluate the energy-efficiency in several antenna selection OFDM systems via simulation results. The simulation parameters are listed in Table IV. The IEEE 802.11n channel model (channel model B) [40] is adopted in our simulations. This channel model has 9 Rayleigh fading paths and is based on measurements of non-line-of-sight (NLOS) environments.

\section{A. Energy Efficiency versus Transmit Power}

We first consider antenna selection MIMO-OFDM systems with $n_{T}=4, n_{R}=1$, and the maximum SNR criterion. The number of equipped RF chains in both combined selection and adaptive selection is $n_{R F}=3$. Fig. 4 shows the energy-efficiency versus the total transmit power $P_{T}$. 


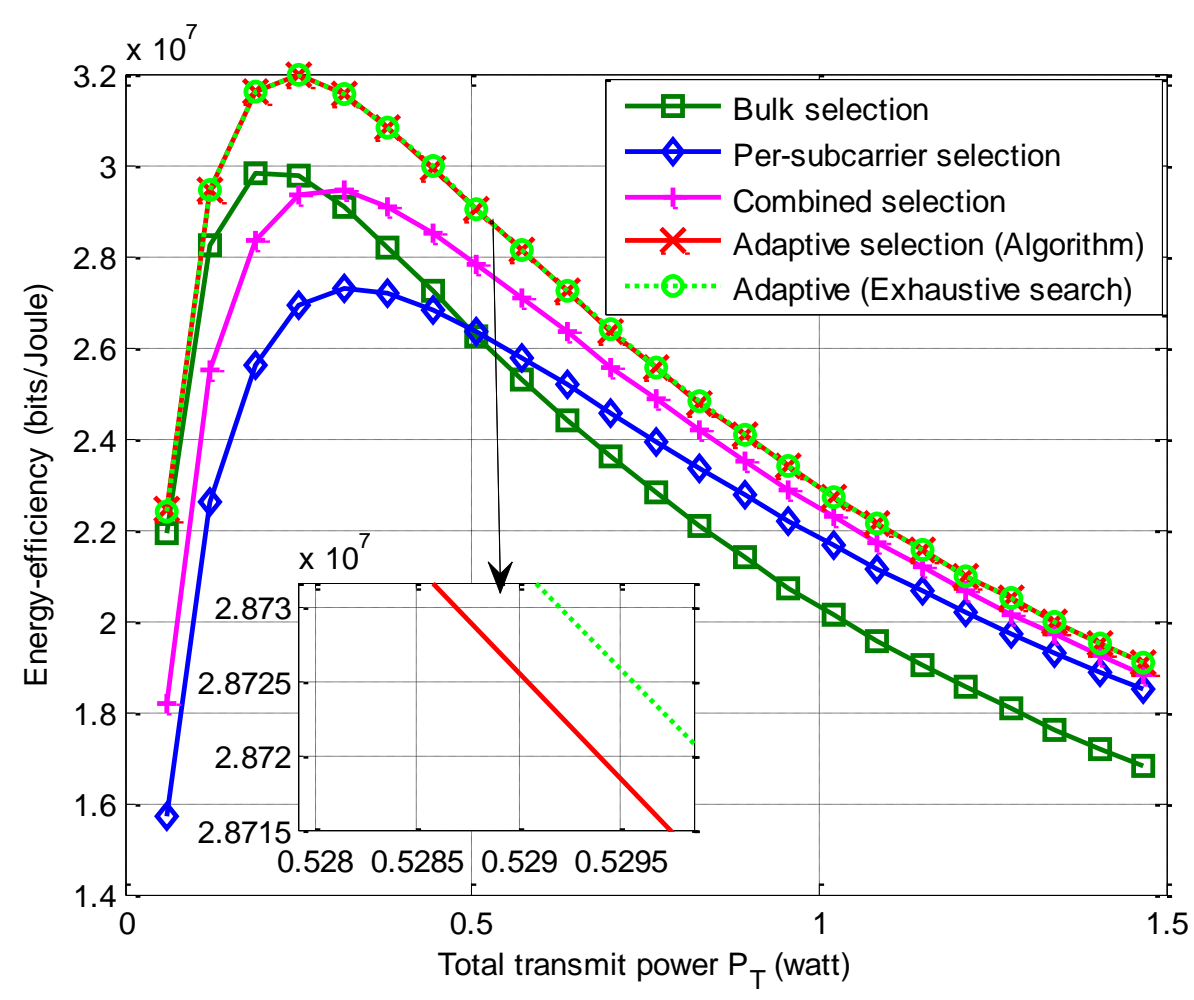

Fig. 4. Energy-efficiency of different antenna selection schemes $\left(n_{T}=4, n_{R}=1\right)$.

The obtained results demonstrate the following. First, the proposed adaptive antenna selection method achieves a better energy-efficiency performance than its counterparts. This comes from the fact that the proposed method can adapt the number of active RF chains $n_{o n}$ according to the channel condition to achieve the maximal $E E$ value. Recall that the numbers of active RF chains in the conventional schemes are fixed. Second, the EE value attained based on the proposed lowcomplexity algorithm is close to that with the exhaustive search method, which demonstrates the effectiveness of this algorithm from a practical viewpoint. Similar observations can be made in the systems with $n_{R}=2$ receive antennas, as shown in Fig. 5. It is also worth noting that there exists the total transmit power $P_{T}$ such that $E E$ is maximized. In Appendix C, we provide an extended discussion regarding this issue.

To have an insight into the adaptive mechanism of the proposed selection approach, we plot in Fig. 6 the numbers of channel realizations that the numbers of active RF chains equal to one, two, and three, when running a simulation with a total of $10^{5}$ channel realizations. It can be seen that when the transmit power $P_{T}$ increases, a larger number of active antennas (i.e., number of active RF chains) is likely selected to attain the maximal $E E$ value. For example, when $P_{T}=0.577 \mathrm{~W}$, the percentage of selection of $n_{o n}=1, n_{o n}=2$, and $n_{o n}=3$, are about $10 \%, 66 \%$, and $24 \%$, respectively. Meanwhile, the corresponding numbers at $P_{T}=2.497 \mathrm{~W}$ are about $1 \%\left(n_{o n}=1\right), 34 \%\left(n_{o n}=2\right)$, and $65 \%\left(n_{\text {on }}=3\right)$. 


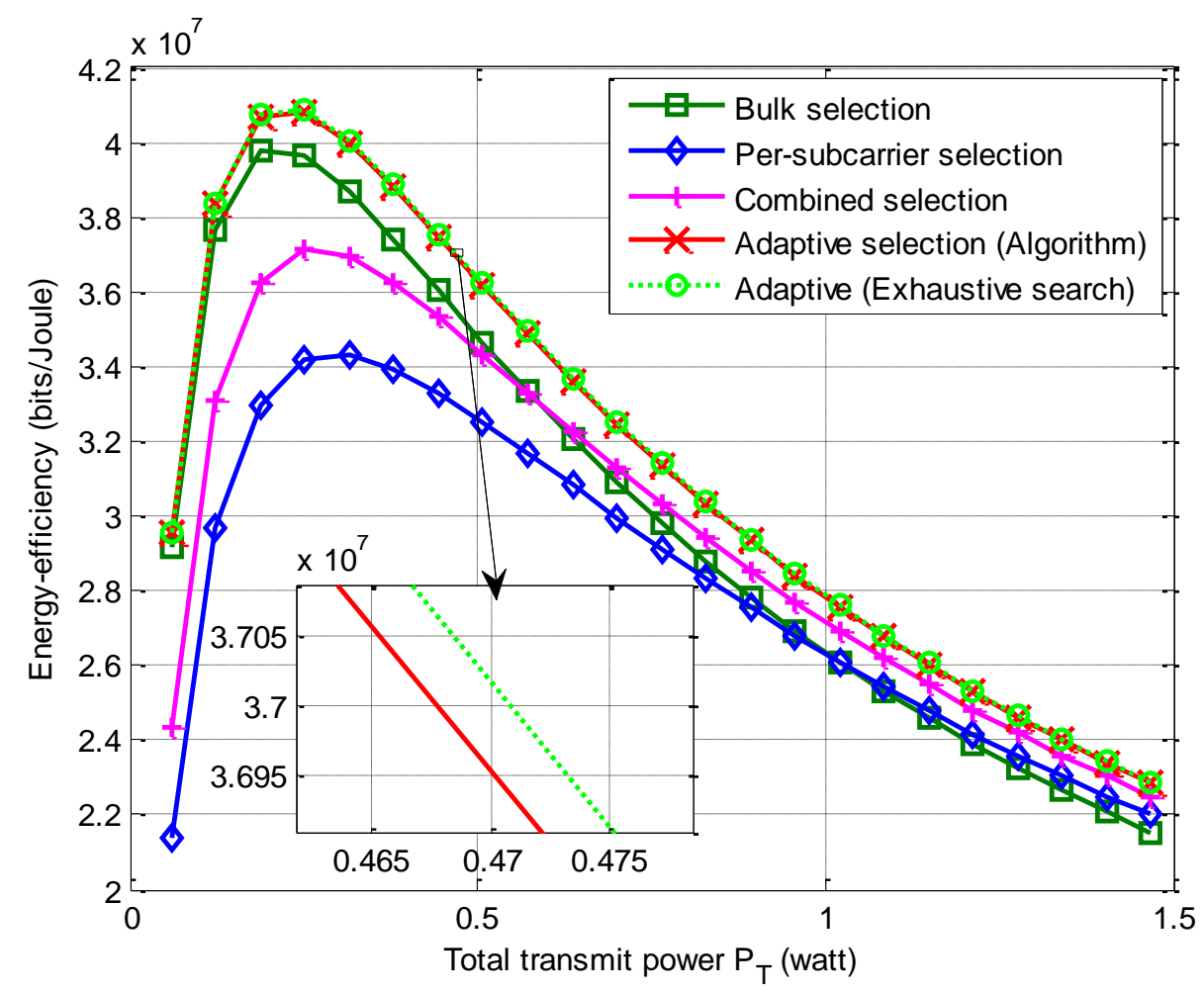

Fig. 5. Energy-efficiency of different antenna selection schemes with two receive antennas $\left(n_{T}=4, n_{R}=2\right)$.

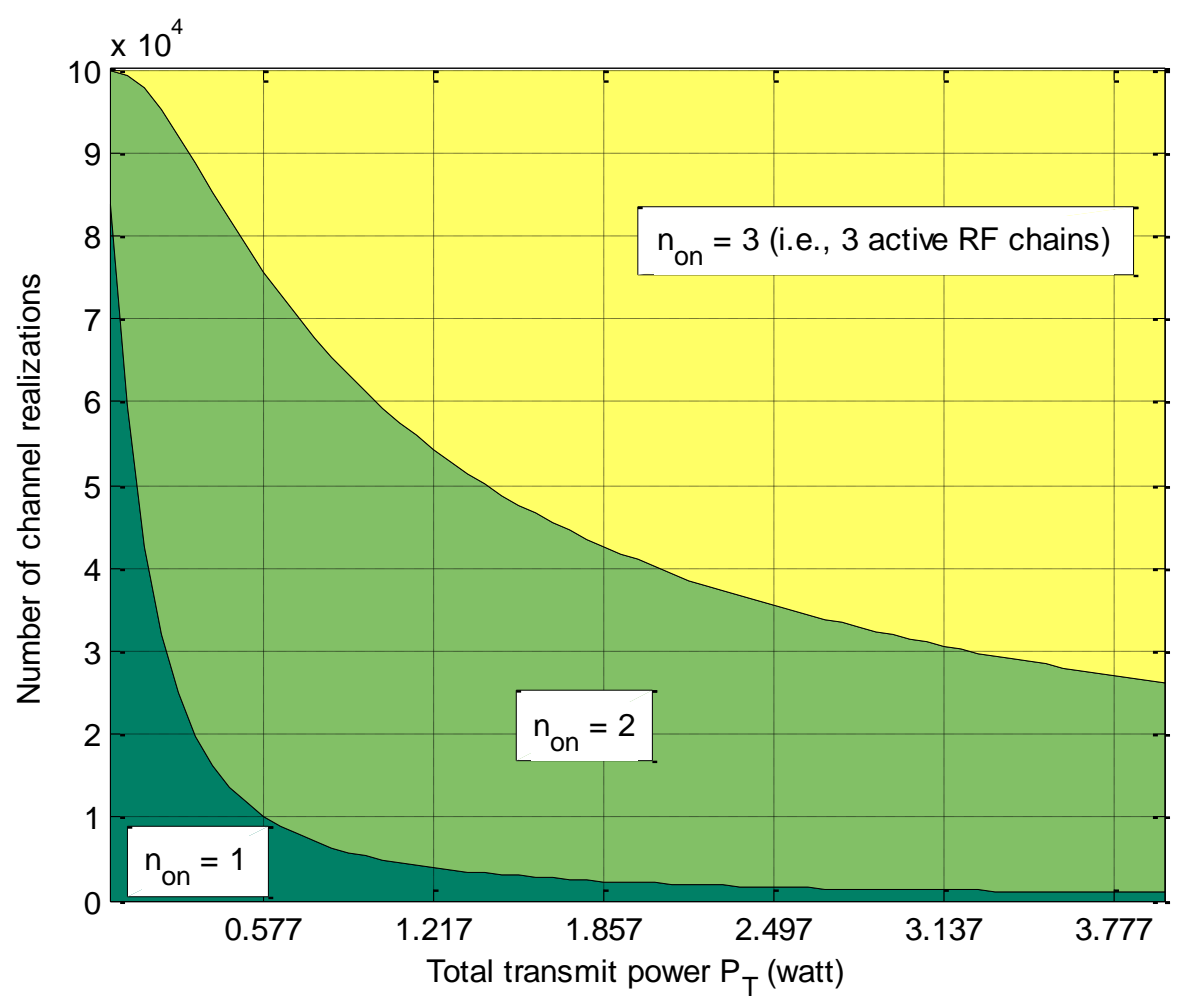

Fig. 6. Number of active RF chains $n_{\text {on }}$ in the adaptive selection scheme $\left(n_{T}=4, n_{R F}=3, n_{R}=1\right)$. 


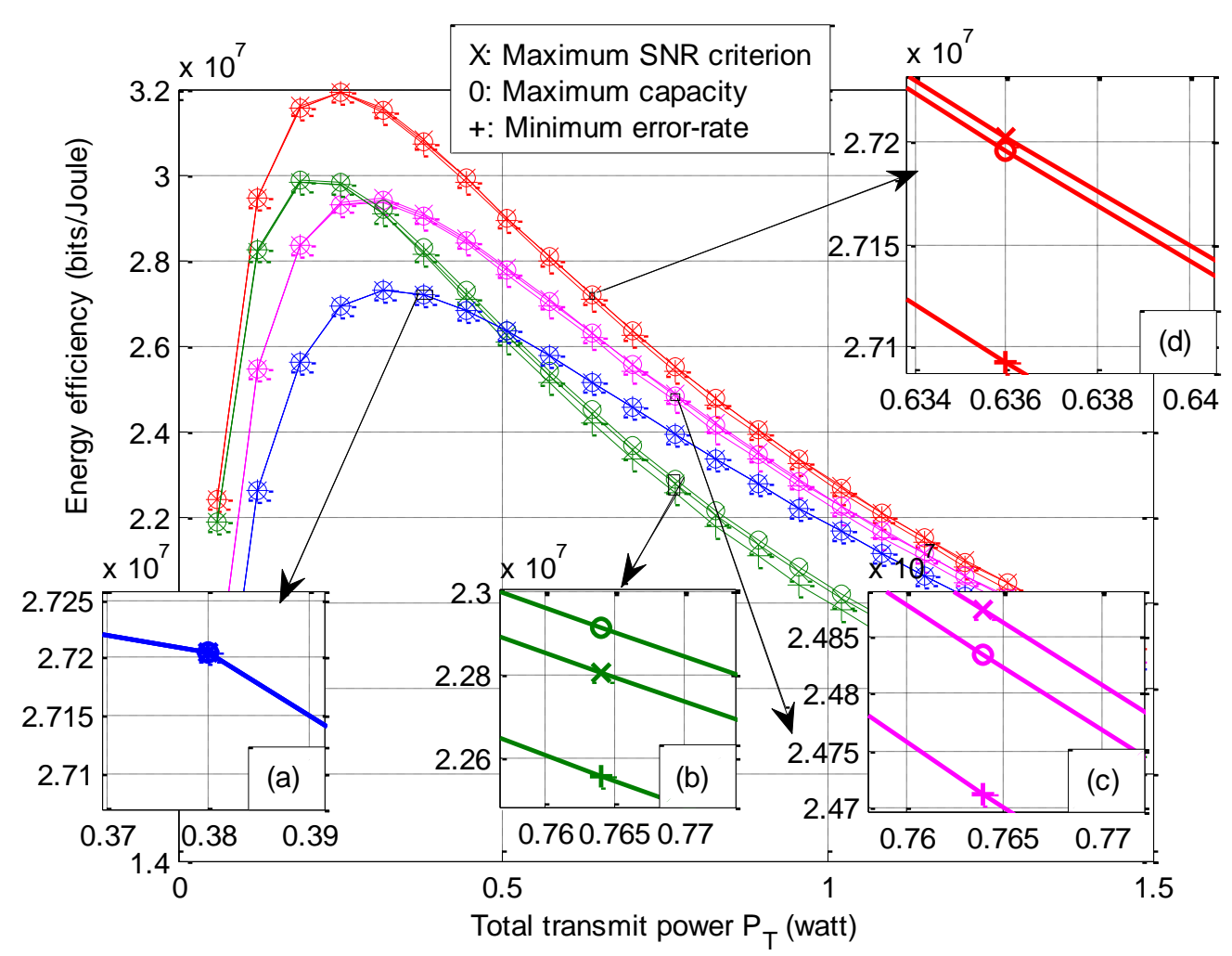

Fig. 7. Energy efficiency under different antenna selection criteria: (a): Per-subcarrier selection; (b): Bulk selection; (c): Combined selection; (d): Adaptive selection.

\section{B. Energy Efficiency under Different Antenna Selection Criteria}

Fig. 7 shows the energy efficiency in the conventional and proposed selection systems under different antenna selection criteria. Three criteria, namely maximum SNR, maximum capacity, and minimum error-rate, introduced in (11) are considered. The results show that in the per-subcarrier selection scheme, all the selection criteria achieve the same energy efficiency. This is due to the fact that antennas are selected independent for each subcarrier in this case. Moreover, at any subcarrier, the selected antenna for the maximum SNR is the one that attains the maximum capacity and minimum error-rate (cf. (5), (11), (12)). For the bulk selection scheme, the maximum capacity criterion achieves the largest energy efficiency. This is because this selection criterion directly maximizes the accumulated capacity across subcarriers, which in turn maximizes energy efficiency (cf. (10)). In the combined and adaptive selection schemes, the maximum SNR criterion can attain higher energy efficiency compared to its counterparts. However, it can be seen that the difference in energy efficiency between the selection criteria is quite small.

\section{Energy Efficiency versus the Number of Transmit Antennas}

Fig. 8 shows the energy efficiency versus the number of equipped transmit antennas in the conventional and proposed systems. It can be seen that, in the bulk selection, combined selection and proposed adaptive selection systems, the $E E$ values increase when the number of antennas $n_{T}$ 


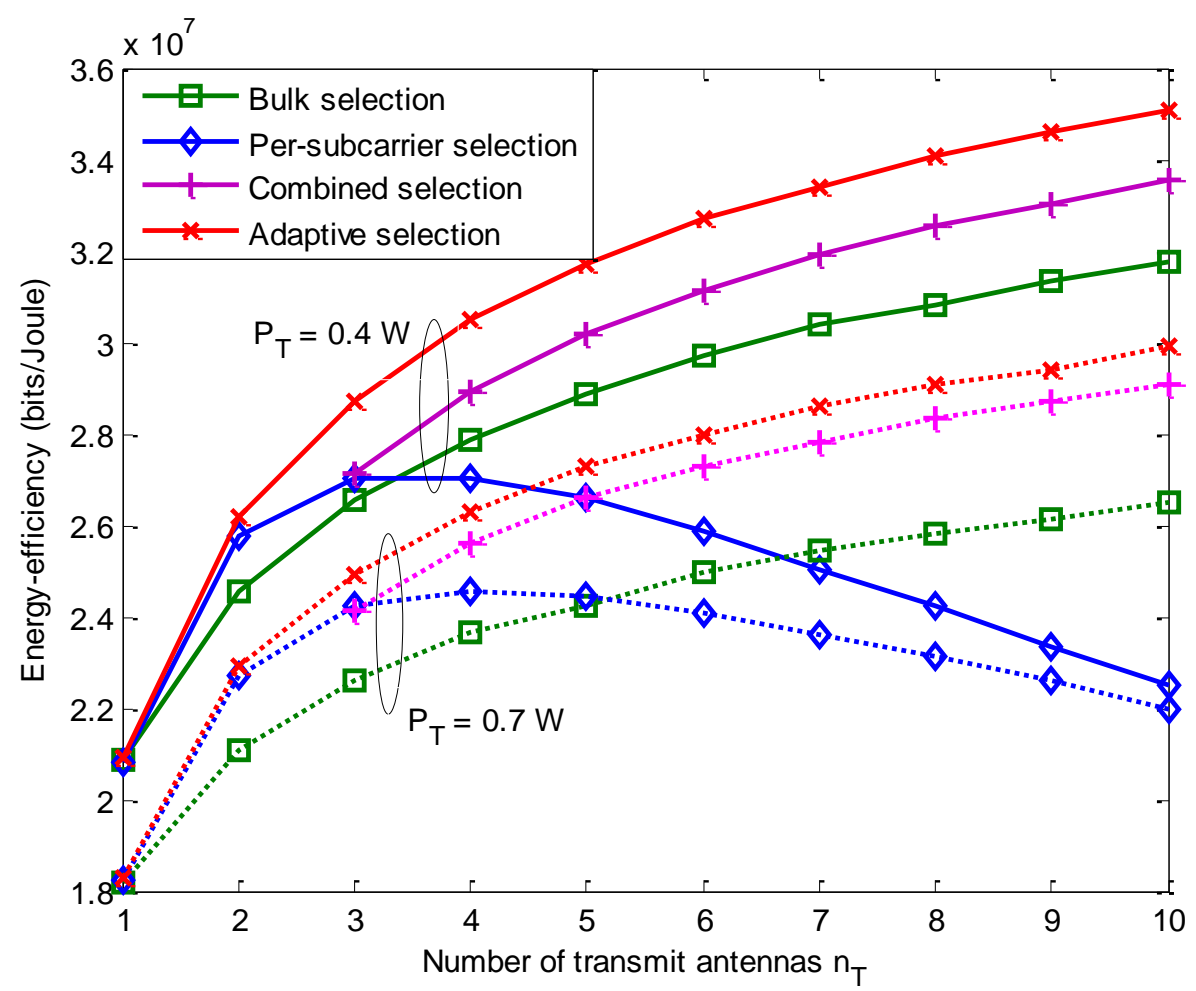

Fig. 8. Energy-efficiency versus the number of transmit antennas $\left(n_{R}=1, n_{R F}=1\right.$ in bulk selection, $n_{R F}=n_{T}$ in per-subcarrier selection, and $n_{R F}=3$ in both combined and adaptive selection schemes).

increases. However, these $E E$ values become saturated when $n_{T}$ becomes very large. This is because the ergodic capacity in antenna selection systems is a logarithmically increasing function w.r.t. $n_{T}$ [41]. Meanwhile, in the per-subcarrier selection system, the $E E$ value first increases and then decreases. This behavior can be explained by the fact that, when $n_{T}$ becomes large, the increased power consumption due to the RF chains has more impact on the energy efficiency than the capacity improvement does, which reduces the $E E$ value (cf.(10)). In addition, we note that an $E E$ comparison among the selection schemes w.r.t. $n_{T}$ depends on particular values of the transmit power and power consumed by hardware. For example, bulk selection is better than per-subcarrier selection when $n_{T}>3$ at $P_{T}=0.4 \mathrm{~W}$, and when $n_{T}>5$ at $P_{T}=0.7 \mathrm{~W}$. It is also important to note that the proposed adaptive selection system outperforms its counterparts for all values of $n_{T}$.

\section{Energy Efficiency versus Spectral Efficiency}

We now examine the EE-SE trade-off in antenna selection MIMO-OFDM systems. The achieved energy efficiency (bits/Joule) versus spectral efficiency (bits/s/Hz) in the systems with $n_{R}=1$ and $n_{R}=2$ are shown in Fig. 9 and Fig. 10, respectively. From an energy-efficiency perspective, it can be seen that bulk selection is effective in the low-SE regime. Meanwhile, per-subcarrier selection and combined selection are suitable in the high-SE and medium-to-high-SE regimes, respectively. Moreover, it can be seen that by adaptively selecting the number of active RF chains, the proposed selection scheme achieves a better the EE-SE trade-off performance compared to the existing 


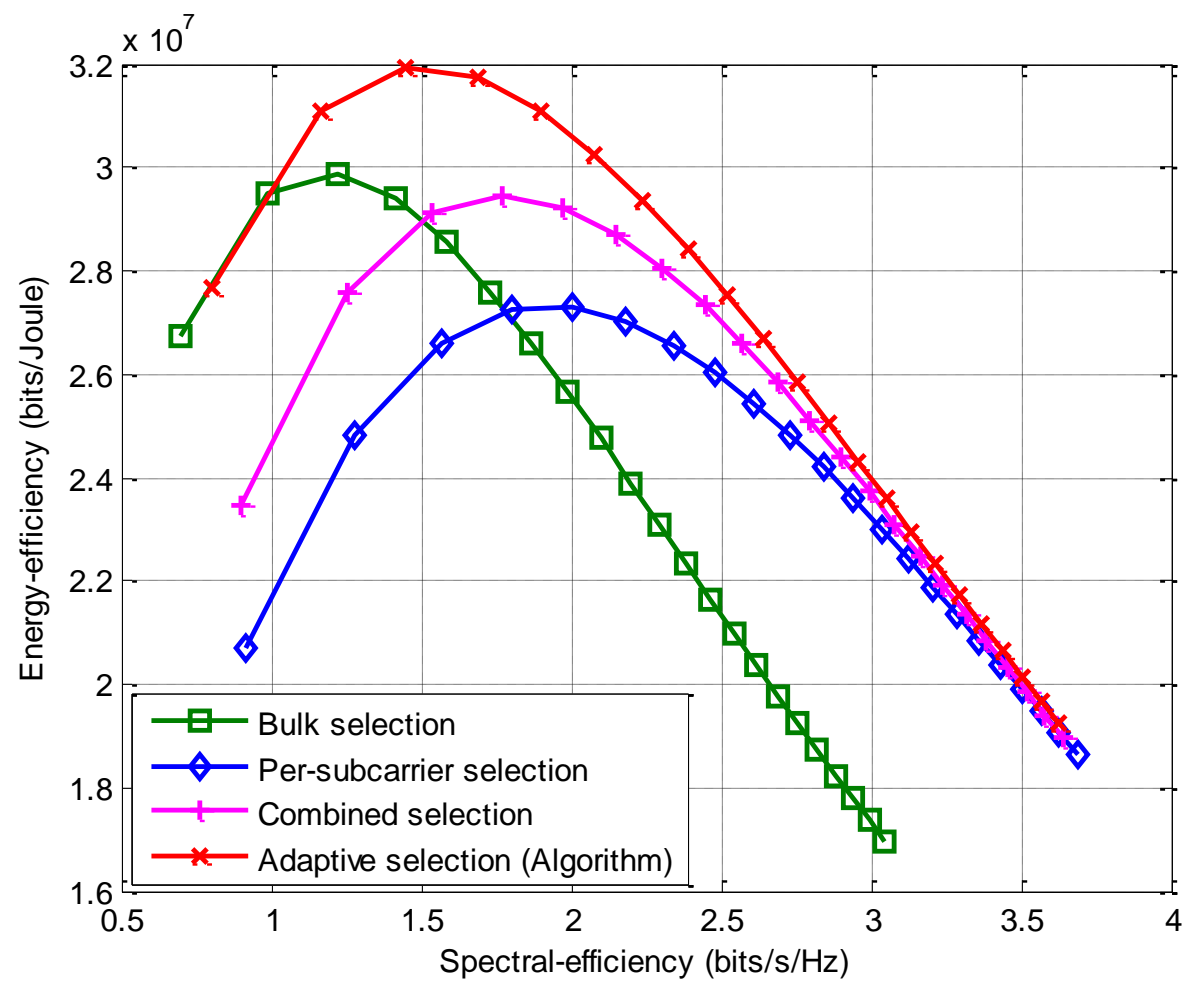

Fig. 9. Energy-efficiency versus spectral-efficiency in different antenna selection schemes $\left(n_{T}=4, n_{R}=1\right)$.

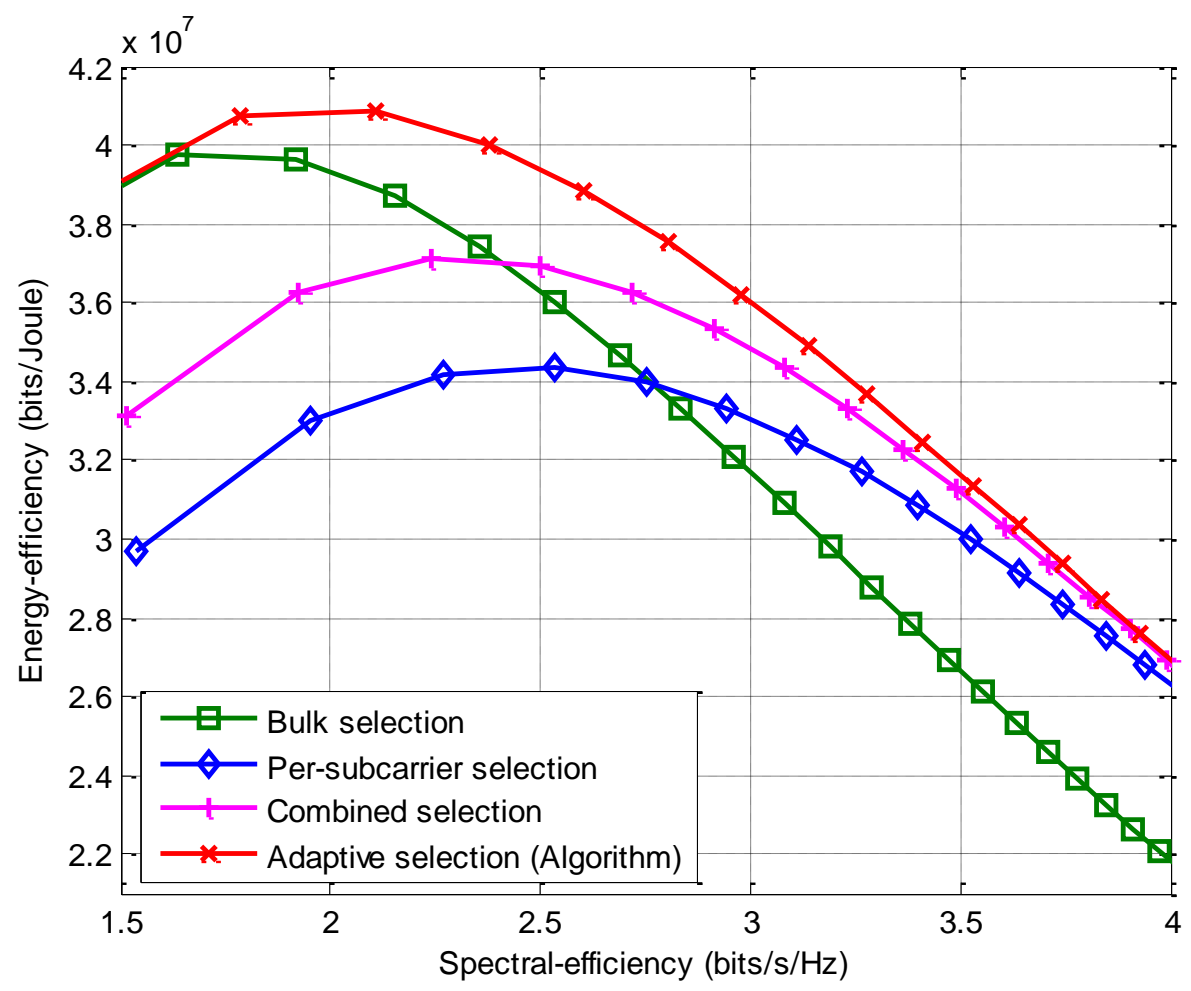

Fig. 10. Energy-efficiency versus spectral-efficiency with two receive antennas $\left(n_{T}=4, n_{R}=2\right)$. 


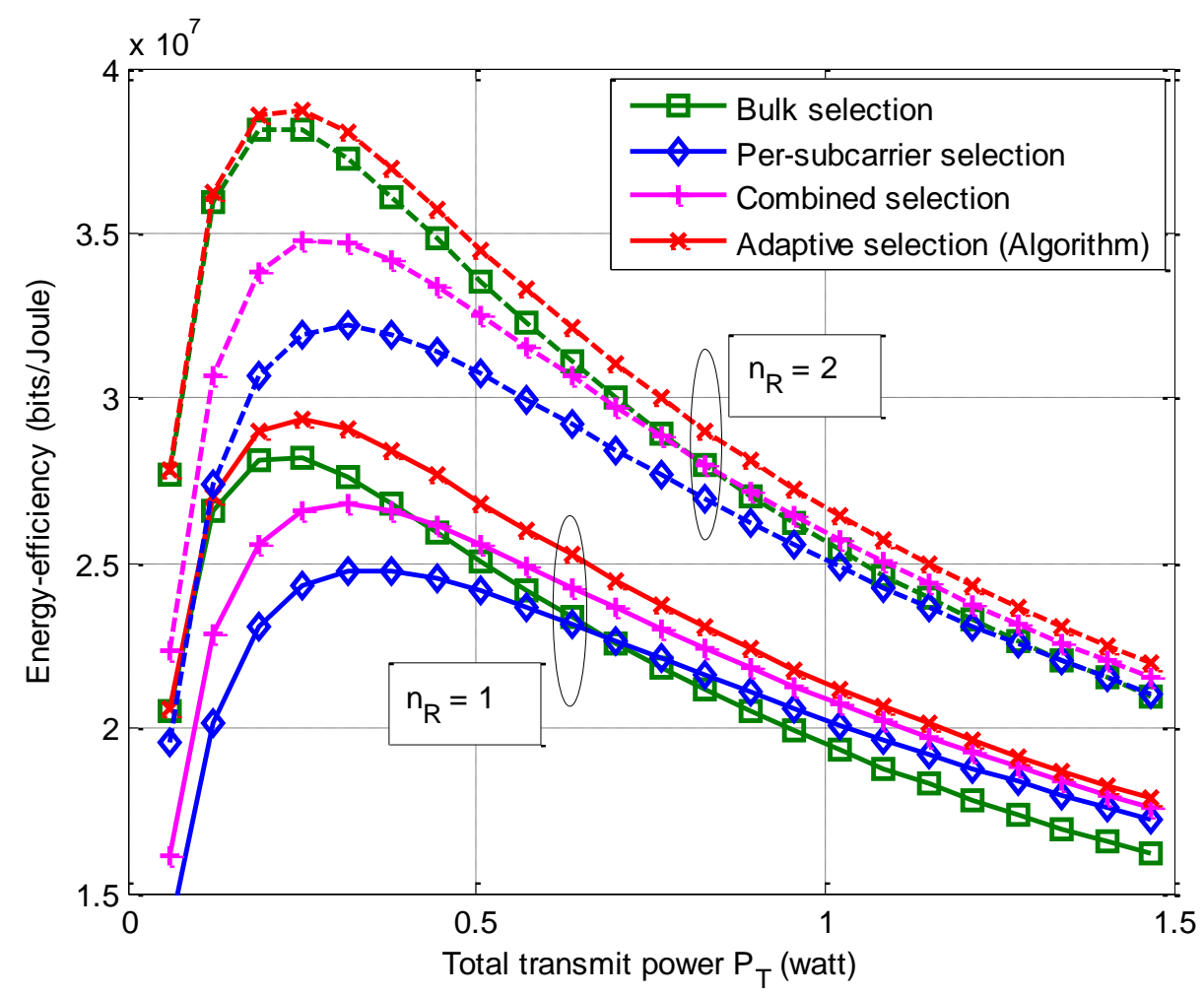

Fig. 11. Energy-efficiency under spatially correlated channels (correlation coefficient of 0.7).

selection schemes. Note that the behavior of these EE-SE curves can be explained from the case of $E E-P_{T}$ curves (e.g., in Fig. 4) given that increasing the spectral efficiency is typically associated with the increasing of the transmit power.

\section{E. Impact of Spatial Correlation on Energy Efficiency}

We next consider the impact of spatial correlation at the transmitter on energy-efficiency in the conventional and proposed systems. The spatially correlated channel is modeled using the Kronecker model [42], i.e., $\mathbf{H}=\mathbf{R}_{R}^{1 / 2} \mathbf{H}_{i i d} \mathbf{R}_{T}^{1 / 2}$, where $\mathbf{R}_{T}$ and $\mathbf{R}_{R}$ are the $n_{T} \times n_{T}$ transmit and the $n_{R} \times n_{R}$ receive correlation matrices, respectively, and $\mathbf{H}_{i i d}$ denotes the $n_{R} \times n_{T}$ channel matrix consisting of independent channel realizations. The achieved energy efficiency is shown in Fig. 11. It can be seen from Fig. 4, Fig. 5, and Fig. 11 that the presence of spatial correlation reduces the energy-efficiency. This makes sense as, given a fixed number of antennas, the correlation between transmit antennas reduces the system capacity, which in turn lowers the energy-efficiency (cf. (10)). However, it is important to note that the proposed system remains superior with respect to energyefficiency compared to the conventional counterparts.

\section{F. Efficacy of Power Loading on Energy Efficiency}

We finally examine the effectiveness of power loading across subcarriers on the energy efficiency. In the simulations, we assume that all subcarriers have an identical spectral mask 


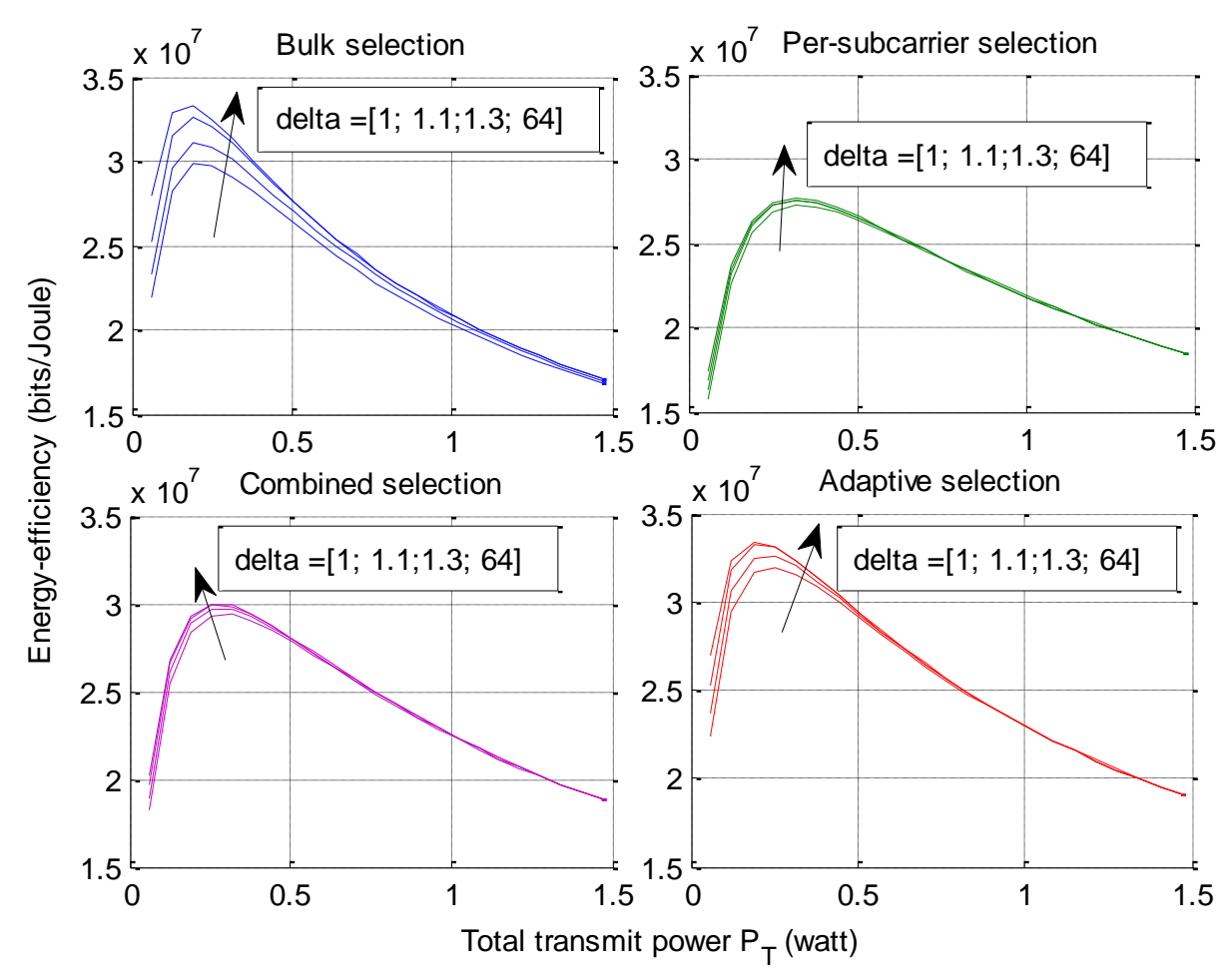

Fig. 12. Energy-efficiency of different antenna selection schemes with power loading $\left(n_{T}=4, n_{R}=1\right)$. Notes: 'delta $=1$ ': equal allocation; 'delta $=64$ ': no spectral mask constraint.

constraint, i.e., $P_{k}^{\text {mask }}=P^{\text {mask }}, \forall k$, for simplicity. Fig. 12 shows the $E E$ values with different levels of spectral mask $\delta$ that is related to $P^{\text {mask }}$ via $P^{\text {mask }}=\delta \times\left(P_{T} / K\right)$. First, it can be seen that power loading offers a better energy-efficiency performance than equal power allocation in all the systems. However, the EE improvement at the high SNR region is marginal. This can be explained by the fact that $E E$ improvement comes from an increase of capacity. Meanwhile, it was shown in [43] that the capacity improvement based on water-filling power allocation (i.e., (36)) is reduced when SNR increases. Therefore, the EE improvement diminishes with an increasing SNR value. The second observation can be made from Fig. 12 is that the $E E$ improvement becomes larger when $\delta$ is larger (i.e., $P^{\text {mask }}$ is higher). However, these $E E$ improvements depend on particular antenna selection schemes. In particular, the $E E$ value is improved quite significantly in bulk-selection and adaptive antenna selection schemes. Meanwhile, in per-subcarrier antenna selection, the $E E$ improvement is marginal. To explain these behaviors, we note that the efficacy of power allocation across subcarriers over equal power allocation comes from a variation of the channel power gains $g_{\widehat{i}_{k}}(k)$ across subcarriers. In per-subcarrier antenna selection, antennas are selected independently for each subcarrier. Thus, it is likely that the difference in the value $c_{\hat{i}_{k}}(k)$ among the selected subcarriers is insignificant in per-subcarrier selection, compared to bulk selection. As a result, power loading is 
not effective in terms of energy-efficiency in the per-subcarrier antenna selection in comparison to the bulk selection and adaptive antenna selection schemes.

\section{CONCLUSIONS}

This paper has investigated the energy-efficiency in MIMO-OFDM systems with different antenna selection approaches. Several important factors that affect the energy-efficiency, including the comparison between the actual transmitted power and the power consumed by the transceiver circuits, types of selection criteria, the number of antennas, and the spatial correlation among antennas, have been examined. The closed-form expressions of the energy efficiency and the EE-SE trade-off in the conventional antenna selection systems have been derived. It is shown that the conventional antenna selection methods exhibit a loss of energy-efficiency. Thus, an adaptive antenna selection method has been proposed to deal with this issue. A greedy algorithm has also been developed to realize a low-complexity adaptive selection scheme. This algorithm can achieve near-optimal performance, which is important when the number of antennas is large. In addition, the energy-efficiency improvement when performing power loading in antenna selection MIMOOFDM systems has been evaluated. Our results show that the proposed adaptive selection scheme outperforms (in terms of the energy-efficiency and the EE-SE trade-off) its counterparts. This work can be extended to multiuser MIMO-OFDMA systems, and we left this for future investigations.

\section{APPENDIX A}

\section{PROOF OF THEOREM 1}

We assume that $\left|h_{j, i}\right|$ follows a Rayleigh distribution with $\varepsilon_{\mathbf{H}}\left\{\left|h_{j, i}\right|^{2}\right\}=1$. It is clear that $g_{i}=\left\|\mathbf{h}_{i}\right\|^{2}$ is a chi-square distribution with $2 n_{R}$ degrees of freedom. Here, the subcarrier index $k$ is dropped for simplicity as subcarriers are assumed independent. The pdf (probability distribution function) and cdf (cumulative distribution function) of $g_{i}$ are given as $f(x)=e^{-x} x^{n_{R}-1} /\left(n_{R}-1\right) !, \forall x>0$, and $F(x)=1-e^{-x} \sum_{v=0}^{n_{R}-1} x^{v} / v !, \forall x>0$, respectively [7]. By using order statistics [44], we can express the pdf of $g_{\hat{i}_{k}}(k)=\left\|\mathbf{h}_{\hat{i}_{k}}(k)\right\|^{2}$ that associated with the selected antennas $\hat{i}_{k}$ at the $k^{\text {th }}$ subcarrier as

$$
\begin{aligned}
f_{M}(x)=n_{T} f(x) F^{n_{T}-1}(x)=n_{T} & \frac{e^{-x} x^{n_{R}-1}}{\left(n_{R}-1\right) !}\left(1-e^{-x} \sum_{v=0}^{n_{R}-1} \frac{x^{v}}{v !}\right)^{n_{T}-1} \\
= & \frac{n_{T}}{\left(n_{R}-1\right) !} e^{-x} x^{n_{R}-1} \sum_{u=0}^{n_{T}-1}\left\{(-1)^{u} \mathrm{C}_{u}^{n_{T}-1} e^{-u x}\left(\sum_{v=0}^{n_{R}-1} \frac{x^{v}}{v !}\right)^{u}\right\},
\end{aligned}
$$

where $\mathrm{C}_{a}^{b}=b ! / a !(b-a)$ ! is the binomial coefficient. By performing a multinomial expansion as $\left(\sum_{v=0}^{n_{R}-1} x^{v} / v !\right)^{u}=\sum_{q=0}^{\left(n_{R}-1\right) u} \alpha_{u, q} x^{q}$, where $\alpha_{u, q}$ is the coefficient resulting from the multinomial 
expansion corresponding to $x^{q}$ (i.e., $\alpha_{u, q}$ is the $q^{\text {th }}$ element of a vector $\boldsymbol{\alpha}_{u}$ that is defined as $\boldsymbol{\alpha}_{0}=1$, $\boldsymbol{\alpha}_{1}=\left[1 / 0 ! 1 / 1 ! 1 / 2 ! \ldots 1 /\left(n_{R}-1\right) !\right]$, and $\boldsymbol{\alpha}_{u}=\boldsymbol{\alpha}_{u-1} \otimes \boldsymbol{\alpha}_{1}$, where $\otimes$ denotes a discrete convolution [45]), we have

$$
f_{M}(x)=\frac{n_{T}}{\left(n_{R}-1\right) !} e^{-x} x^{n_{R}-1} \sum_{u=0}^{n_{T}-1}\left\{(-1)^{u} \mathrm{C}_{u}^{n_{T}-1} e^{-u x} \sum_{q=0}^{\left(n_{R}-1\right) u} \alpha_{u, q} x^{q}\right\} .
$$

The expected value of $\varepsilon_{\mathbf{H}}\left\{\log _{2}\left(1+\bar{\rho} g_{\bar{i}_{k}}(k)\right)\right\}$ can now be calculated as

$$
\begin{aligned}
\varepsilon_{\mathbf{H}}\left\{\log _{2}\left(1+\bar{\rho} g_{\bar{i}_{k}}(k)\right)\right\} & =\int_{0}^{+\infty} \log _{2}(1+\bar{\rho} x) f_{M}(x) d x \\
& =\int_{0}^{+\infty}\left(\log _{2}(1+\bar{\rho} x) \frac{n_{T}}{\left(n_{R}-1\right) !} e^{-x} x^{n_{R}-1} \sum_{u=0}^{n_{T}-1}\left\{(-1)^{u} C_{u}^{n_{T}-1} e^{-u x} \sum_{q=0}^{\left(n_{R}-1\right) u} \alpha_{u, q} x^{q}\right\}\right) d x \\
& =\frac{n_{T}}{\left(n_{R}-1\right) !} \sum_{u=0}^{n_{T}-1}\left\{(-1)^{u} \mathrm{C}_{u}^{n_{T}-1} \sum_{q=0}^{\left(n_{R}-1\right) u} \alpha_{u, q} \int_{0}^{+\infty} \log _{2}(1+\bar{\rho} x) x^{n_{R}+q-1} e^{-(u+1) x} d x\right\}
\end{aligned}
$$

By using the integral result in [46, Eq. (32) \& Eq. (78)], we can express the integral in (39) as

$$
\begin{aligned}
Q & =\int_{0}^{+\infty} \log _{2}(1+\bar{\rho} x) x^{n_{R}+q-1} e^{-(u+1) x} d x \\
& =\frac{\left(n_{R}+q-1\right) !}{\bar{\rho}^{n_{R}+q} \ln 2} e^{(u+1) / \bar{\rho}} \sum_{s=1}^{n_{R}+q}\left(\frac{u+1}{\bar{\rho}}\right)^{-s} \Gamma\left(-n_{R}-q+s, \frac{u+1}{\bar{\rho}}\right),
\end{aligned}
$$

where $\Gamma(a, x)=\int_{x}^{+\infty} e^{-t} t^{a-1} d t$ is the incomplete gamma function [47, Eq. (8.350.2)]. From (16), (39), and (40), we finally arrive at (17).

\section{APPENDIX B}

\section{PROOF OF THEOREM 2}

Let us first denote $w_{i}=\sum_{k=0}^{K-1} g_{i}(k)$. Then, we can explicitly express $w_{i}$ as (cf. (2))

$$
w_{i}=\sum_{k=0}^{K-1} g_{i}(k)=\sum_{k=0}^{K-1}\left\|\mathbf{h}_{i}(k)\right\|^{2}=\sum_{k=0}^{K-1} \sum_{j=1}^{n_{R}}\left|h_{j, i}(k)\right|^{2}
$$

As the channel coefficients $\left|h_{j, i}(k)\right|$ are i.i.d. Rayleigh random variables, it follows that $w_{i}$ in (41) is a chi-square distribution with $2 n_{R} K$ degrees of freedom. Therefore, we can express the pdf and cdf of $w_{i}$ as $f(y)=e^{-y} y^{n_{R} K-1} /\left(n_{R} K-1\right) !, \forall y>0$ and $F(y)=1-e^{-y} \sum_{s=0}^{n_{R} K-1} y^{s} / s !, \forall y>0$ respectively [7]. By performing similar calculations as in Appendix A, we obtain the pdf of $w_{\hat{i}}$ that is corresponding to the selected antenna $\hat{i}, \hat{i}=\arg \max _{i=1, \ldots, n_{T}} w_{i}$ (cf. (14)), as 


$$
f_{N}(y)=\frac{n_{T}}{\left(n_{R} K-1\right) !} e^{-y} y^{n_{R} K-1} \sum_{u=0}^{n_{T}-1}\left\{(-1)^{u} \mathrm{C}_{u}^{n_{T}-1} e^{-u y} \sum_{t=0}^{\left(n_{R} K-1\right) u} \beta_{u, t} y^{t}\right\},
$$

where $\beta_{u, t}$ is the coefficient resulting from the multinomial expansion of $\left(\sum_{s=0}^{n_{R} K-1} y^{s} / s !\right)^{u}$, i.e., $\left(\sum_{s=0}^{n_{R} K-1} y^{s} / s !\right)^{u}=\sum_{t=0}^{\left(n_{R} K-1\right) u} \beta_{u, t} y^{t}$. Consequently, the expected value of $\varepsilon_{\mathbf{H}}\left\{w_{\hat{i}}\right\}$ is calculated as

$$
\begin{aligned}
\varepsilon_{\mathbf{H}}\left\{w_{i}\right\} & =\int_{0}^{+\infty} y f_{N}(y) d y \\
& =\int_{0}^{+\infty}\left(y \frac{n_{T}}{\left(n_{R} K-1\right) !} e^{-y} y^{n_{R} K-1} \sum_{u=0}^{n_{T}-1}\left\{(-1)^{u} \mathrm{C}_{u}^{n_{T}-1} e^{-u y} \sum_{t=0}^{\left(n_{R} K-1\right) u} \beta_{u, t} y^{t}\right\}\right) d y \\
& =\frac{n_{T}}{\left(n_{R} K-1\right) !} \sum_{u=0}^{n_{T}-1}\left\{(-1)^{u} \mathrm{C}_{u}^{n_{T}-1} \sum_{t=0}^{\left(n_{R} K-1\right) u} \beta_{u, t} \int_{0}^{+\infty} y^{n_{R} K+t} e^{-(u+1) y} d y\right\} \\
& =\frac{n_{T}}{\left(n_{R} K-1\right) !} \sum_{u=0}^{n_{T}-1}\left\{(-1)^{u} \mathrm{C}_{u}^{n_{T}-1} \sum_{t=0}^{\left(n_{R} K-1\right) u} \beta_{u, t}\left(n_{R} K+t\right) !(u+1)^{-n_{R} K-t-1}\right\},
\end{aligned}
$$

where, in the last equality, we have used the integral of $\int_{0}^{+\infty} x^{n} e^{-\mu x} d x=n ! \mu^{-n-1}$ [47, Eq. (3.351.3)]. By substituting (43) into (20), we obtain (21). This completes the proof.

\section{APPENDIX C}

\section{Joint Transmit Power Allocation And AdAptive Antenna SELECTION}

It is shown in Fig. 4 and Fig. 5 that it is possible to allocate the total transmit power $P_{T}$ to maximize $E E$. Motivated by this, in this appendix, we further consider a joint power allocation and adaptive antenna selection for the maximum EE under a quality-of-service (QoS) constraint. For simplicity, we restrict ourselves to the system with equal power allocation across subcarriers. The formulated problem is jointly select the number of active RF chains, transmit antenna indices, and the optimal power per subcarrier $P_{t}^{\text {opt }}$ to maximize EE subject to a QoS constraint. Note that the optimal total power per OFDM symbol $P_{T}^{\text {opt }}$ is then obtained as $P_{T}^{\text {opt }}=K P_{t}^{\text {opt }}$.

Let us first consider the optimal transmit power without a QoS constraint. As the instantaneous energy-efficiency $E E\left(P_{t}\right)$ is a pseudo-concave function with respect to $P_{t}$ [18]. The unique maximum value occurs when $\partial E E\left(P_{t}\right) / \partial P_{t}=0$. Specifically, the instantaneous EE can be expressed as (cf. (3), (5), (10))

$$
E E\left(P_{t}\right)=\frac{(W / K) \times \sum_{k=0}^{K-1} \log _{2}\left(1+P_{t} g_{\widehat{i}_{k}}(k) / \sigma_{n}^{2}\right)}{K P_{t} / \eta+n_{o n} P_{c t x}+n_{R} P_{c r x}+P_{b b}} .
$$


Thus, an equation of $\partial E E\left(P_{t}\right) / \partial P_{t}=0$ is equivalent to

$$
\sum_{k=0}^{K-1} \log _{2}\left(1+P_{t} g_{\hat{i}_{k}}(k) / \sigma_{n}^{2}\right)-\left(P_{t}+\eta\left(n_{o n} P_{c t x}+n_{R} P_{c r x}+P_{b b}\right) / K\right) \times \sum_{k=0}^{K-1} \frac{g_{\hat{i}_{k}}(k) / \sigma_{n}^{2}}{\left(1+P_{t} g_{\hat{i}_{k}}(k) / \sigma_{n}^{2}\right) \ln 2}=0 .
$$

It is hard to obtain a closed-form solution for (45). However, numerical methods can be used to obtain the optimal power value $P_{t}^{*}$.

In systems with SNR constraint, it is required that $\Omega \geq \gamma_{t h}$, where $\Omega$ is the received SNR and $\gamma_{t h}$ is the required SNR threshold. In multicarrier transmissions, each subcarrier has a different channel gain in general. To facilitate a power allocation problem, the average channel power gain (or average SNR) over frequency domain is often used in the literature [48]. The average SNR over all the subcarriers in antenna selection MIMO-OFDM systems can be expressed as (cf. (3))

$$
\Omega=\frac{P_{t}}{\sigma_{n}^{2} K} \sum_{k=0}^{K-1} g_{\hat{i}_{k}}(k):=P_{t} \psi
$$

where a parameter $\psi$ is defined as

$$
\psi:=\frac{1}{\sigma_{n}^{2} K} \sum_{k=0}^{K-1} g_{\hat{i}_{k}}(k)
$$

As the SNR constraint requires that $P_{t} \psi \geq \gamma_{t h}$, the optimal transmit power can be determined as [18]

$$
P_{t}^{o p t}= \begin{cases}P_{t}^{*}, & \text { if } \psi P_{t}^{*} \geq \gamma_{t h}, \\ \gamma_{t h} / \psi, & \text { if } \psi P_{t}^{*}<\gamma_{t h}\end{cases}
$$

Thus, a joint power allocation and adaptive antenna selection subject to SNR constraint can be realized by inserting the following steps between Step (9) and Step (10) in the algorithm in Table I.

Step a) Calculate the power $P_{t}^{*}$ by solving (45).

Step b) Calculate the parameter $\psi$ based on (47).

Step c) Determine the optimal transmit power $P_{t}^{\text {opt }}$ via (48).

\section{REFERENCES}

[1] A. Fehske, G. Fettweis, J. Malmodin, and G. Biczok, "The global footprint of mobile communications: The ecological and economic perspective," IEEE Communications Magazine, vol. 49, no. 8, pp. 55- 62, Aug. 2011.

[2] G. Y. Li et al., "Energy-efficient wireless communications: Tutorial, survey, and open issues," IEEE Wireless commun., vol. 18, no. 6, pp. 28-35, Dec. 2011.

[3] L. M Correia et al., "Challenges and enabling technologies for energy aware mobile radio networks," IEEE Communications Magazine, vol. 48, no.11, pp. 66-72, Nov. 2010.

[4] J. Joung, C. K. Ho, K. Adachi, and S. Sun, "A survey on power-amplifier-centric techniques for spectrum and energy efficient wireless communications,” IEEE Commun. Surveys \& Tutorials, Aug. 2014 (early access articles). 
[5] G. L. Stuber et al., "Broadband MIMO-OFDM wireless communications," The proceedings of the IEEE, vol. 92, no. 2, pp. 271-294, Feb. 2004.

[6] L. Hanzo, Y. Akhtman, M. Jiang, and L. Wang, MIMO-OFDM for LTE, WIFI and WIMAX: Coherent versus NonCoherent and Cooperative Turbo-Transceivers, Wiley-IEEE Press, 2011.

[7] D. Tse and P. Viswanath, Fundamentals of Wireless Communication, Cambridge University Press, USA, 2005.

[8] A. F. Molisch and M. Z. Win, "MIMO systems with antenna selection," IEEE Microwave Magazine, vol. 5, no. 1, pp. 45-56, March 2004.

[9] C. M. Vithanage, J. P. Coon, and S. C. J. Parker, “On capacity-optimal precoding for multiple antenna systems subject to EIRP restrictions," IEEE Trans. Wireless Commun., vol. 7, no. 12, pp. 5182-87, Dec. 2008.

[10] N. B. Mehta, A. F. Molisch, and S. Kashyap, “Antenna selection in LTE: from motivation to specification,” IEEE Communications Magazine, vol. 50, no. 10, pp. 144-150, Oct. 2012.

[11] H. Zhang and R. U. Nabar, "Transmit antenna selection in MIMO-OFDM systems: bulk versus per-tone selection,” in Proc. IEEE Intern. Conf. Commun., ICC 2008, pp. 4371-4375, May 2008.

[12] H. Zhang, A. F. Molisch, and J. Zhang, “Appling antenna selection in WLAN for achieving broadband multimedia communications," IEEE Transactions on Broadcasting, vol. 52, no. 4, pp. 475-482, Dec. 2006.

[13] Z. Tang, H. Suzuki, and I. B. Collings, "Performance of antenna selection for MIMO-OFDM systems based on measured indoor correlated frequency selective channels," in Proc. ATNAC, pp. 435-439, Dec. 2006.

[14] M. Sandell and J. Coon, "Per-subcarrier antenna selection with power constraints in OFDM systems," IEEE Transactions on Wireless Communications, vol. 8, no. 2, pp. 673-677, Feb. 2009.

[15] J. P. Coon and M. Sandell, "Combined bulk and per-tone transmit antenna selection in OFDM systems", IEEE Comm. Letters, vol. 14, no. 5, pp. 426-428, May 2010.

[16] M. Sandell and J. Coon, "Performance of combined bulk and per-tone antenna selection precoding in coded OFDM systems,” IEEE Trans. Commun., vol. 60, no. 3, pp. 655-660, March 2012.

[17] J. P. Coon and M. Sandell, "Performance of transmit antenna selection in space-time-coded OFDM systems", IEEE Transactions on Vehicular Technology, vol. 60, no. 6, pp. 2824-2828, July 2011.

[18] C. Jiang and L. J. Cimini, "Antenna selection for energy-efficient MIMO transmission," IEEE Wireless Communications Letters, vol. 1, no. 6, pp. 577-580, Dec. 2012.

[19] X. Zhou, B. Bai, and W. Chen, "Iterative antenna selection for multi-stream MIMO under a holistic power model," IEEE Wireless Communications Letters, vol. 3, no. 1, pp. 82-85, Feb. 2014.

[20] H. Li, L. Song, and M. Debbah, "Energy efficiency of large-scale multiple antenna systems with transmit antenna selection," IEEE Trans. Commun., vol. 62, no. 2, pp. 638-647, Feb. 2014.

[21] J. Joung, Y. K. Chia, and S. Sun, "Energy-efficient, large-scale distributed-antenna system (L-DAS) for multiple users," IEEE Journal of Selected Topics in Signal Processing, vol. 8, no. 5, pp. 954-965, Oct. 2014.

[22] E. Eraslan, C. Y. Wang, and B. Daneshrad, "Practical energy-aware link adaptation for MIMO-OFDM systems," IEEE Trans. on Wireless Commun., vol. 13, no. 1, pp. 246-258, Jan. 2014.

[23] X. Ge et al., "Energy-efficiency optimization for MIMO-OFDM mobile multimedia communication systems with QoS constraints,” IEEE Trans. Veh. Technol., vol. 63, no. 5, pp. 2127-2138, June 2014.

[24] N. P. Le, L. C. Tran, and F. Safaei, "Energy-efficiency analysis of per-subcarrier antenna selection with peakpower reduction in MIMO-OFDM wireless systems," Int. Journal of Antennas and Propagation 2014.

[25] D. J. Love, "On the probability of error of antenna-subset selection with space-time block codes," IEEE Trans. Commun., vol. 53, no. 11, Nov. 2005. 
[26] Z. Chen, J. Yuan, and B. Vucetic, "Analysis of transmit antenna selection/maximal-ratio combining in Rayleigh fading channels," IEEE Trans. Veh. Technol., vol. 54, no. 4, pp. 1312-1321, July 2005.

[27] Y. (G.) Li, N. Seshadri, and S. Ariyavisitakul, "Channel estimation for OFDM systems with transmitter diversity in mobile wireless channels," IEEE J. Select. Areas Commun., vol. 17, pp. 461-471, March 1999.

[28] N. B. Mehta, A. F. Molisch, J. Zhang, and E. Bala, “Antenna selection training in MIMO-OFDM/OFDMA cellular systems," in Proc. IEEE CAMSAP 2007, pp. 113-116, Dec. 2007.

[29] M. Sandell and J. Coon, "Joint data detection and channel sounding for TDD systems with antenna selection," in Proc. IEEE Vehicular Tech. Conf., VTC-Spring 2011, pp. 1-5, May 2011.

[30] H. Bolcskei, D. Gesbert, and A. J. Paulraj, "On the capacity of OFDM based spatial multiplexing systems," IEEE Transactions on Communications, vol. 50, no. 2, pp. 225-234, Feb. 2002.

[31] S. Cui, A. Goldsmith, and A. Bahai, "Energy-constrained modulation optimization," IEEE Transactions on Wireless Communications, vol. 4, no. 5, pp. 2349-2360, Sept. 2005.

[32] S. C. Cripps, RF Power Amplifiers for Wireless Communications, Artech House, 2006.

[33] F. Wang et al., "Design of wide-bandwidth envelope-tracking power amplifiers for OFDM applications," IEEE Trans. Microwave Theory and Techniques, vol. 53, no. 4, pp. 1312-1321, April 2005.

[34] K. Cho and D. Yoon, "On the general BER expression of one-and two-dimensional amplitude modulations," IEEE Trans. Commun., vol. 50, no. 7, pp. 1074 -1080, Nov. 2002.

[35] J. Jiang et al., "On the relation between energy efficiency and spectral efficiency of multiple-antenna systems," IEEE Trans. on Veh. Tech., vol. 62, no. 7, pp. 3463-3469, Sept. 2013.

[36] A. Batra et al., "Multiband OFDM physical layer specification," WiMedia Alliance, Release 1.5, August 2009.

[37] A. Leke and J. Cioffi, "A maximum rate loading algorithm for discrete multitone modulation systems," in Proc. IEEE Global Telecommunications Conf., Globecom 1997, pp. 1514-1518, Nov. 1997.

[38] S. Boyd and L. Vandenberghe, Convex Optimization, Cambridge University Press, 2004.

[39] X. Ling, B. Wu, P. H. Ho, F. Luo, and L. Pan, "Fast water-filling for agile power allocation in multi-channel wireless communications," IEEE Communications Letters, vol. 16, no. 8, pp. 1212-1215, August 2012.

[40] V. Erceg et al., “TGn Channel Models,” IEEE 802.11-03/940r4, May 2004.

[41] S. Sanayei and A. Nosratinia, "Capacity of MIMO channels with antenna selection", IEEE Transactions on Information Theory, vol. 53, no. 11, pp. 4356-4362, Nov. 2007.

[42] A. Paulraj, D. Gore, and R. Nabar, Introduction to Space-Time Wireless Communications, Cambridge University Press, 2003.

[43] H. Moon, "Water-filling power allocation at high SNR regimes," IEEE Trans. Commun., vol. 59, no. 3, pp. 708715, March 2011.

[44] H. A. David, Order Statistics, John Wiley \& Sons, 1981.

[45] M. Lari, A. Mohammadi, A. Abdolali, and I. Lee, "Characterization of effective capacity in antenna selection MIMO systems,” KICS/IEEE Journal of Commun. and Networks, vol. 15, no. 5, pp. 476-485, Oct. 2013.

[46] M. S. Alouini and A. J. Goldsmith, "Capacity of Rayleigh fading channels under different adaptive transmission and diversity-combining techniques," IEEE Trans. on Veh. Tech., vol. 48, no. 4, pp. 1165-1181, July 1999.

[47] I. S. Gradshteyn and I. M. Ryzhik, Table of Integrals, Series and Products, Academic Press, 2007.

[48] S. V. Hanly, L. L. H. Andrew, and T. Thanabalasingham, "Dynamic allocation of subcarriers and transmit powers in an OFDMA cellular network," IEEE Trans. on Infor. Theory, vol. 55, no. 12, pp. 5445-5462, Dec. 2009. 